\title{
Nonlinear Impairment Compensation using Backpropagation
}

\author{
Ezra Ip ${ }^{1}$ and Joseph M. Kahn² \\ ${ }^{1}$ NEC Labs America, Princeton, NJ, USA \\ 2Stanford University, Stanford, CA, USA
}

\section{Introduction}

The ultimate information capacity of fiber is limited by the Kerr nonlinearity, wherein the refractive index of the waveguide changes in proportion to the signal intensity. Nonlinearity makes it impossible to achieve an arbitrarily high capacity by increasing the launched power, as nonlinear distortion ultimately grows faster than the signal-to-noise ratio (SNR). At low launched power, system performance is limited by additive white Gaussian noise (AWGN), so the capacity increases with power. At high power, however, nonlinear effects dominate. For a given channel, there exists an optimum launched power and ultimate capacity that are dependent on the system design parameters. The capacity of fiber has been studied in (Mitra \& Stark, 2001) and (Essiambre et al., 2008).

Recently, coherent optical receivers have begun to approach technological maturity. In a coherent receiver, the optical signal is downconverted to the electronic domain, preserving all relevant information on the optical electric fields in the two polarizations. The baseband equivalent electrical signals can be sampled and processed by a digital signal processor (DSP), enabling software algorithms to compensate channel impairments (Ip, et al. 2008).

Various methods of compensating nonlinearity have been proposed. It was shown in (Ho \& Kahn, 2004) that in zero-dispersion fiber, interaction between nonlinearity and noise, known as nonlinear phase noise (NLPN), causes the received constellation to be spiral-shaped. A receiver can partially compensate NLPN by either de-rotating the received signal by a phase proportional to the received power, or by using modified decision boundaries. Improvement in system performance by 0.5 to $1 \mathrm{~dB}$ has been observed in submarine systems with low local dispersion and near-perfect dispersion compensation per span (Charlet et al., 2006).

Nonlinear phase rotation has also been used in orthogonal frequency-division multiplexing (OFDM) systems, where it is called self-phase modulation compensation (SPMC). In (Lowery, 2007a), transmitter-side SPMC was proposed, while (Lowery, 2007b) showed that further performance improvement can be obtained by splitting the SPMC between the transmitter and receiver. An investigation of the performance dependence on the dispersion map was carried out in (Liu \& Tkach, 2009).

Signal propagation is ultimately governed by a nonlinear Schrödinger equation (NLSE). We shall see later that the efficacy of a nonlinear compensation algorithm depends on how 
accurately it estimates the optical signal amplitude at each point in the fiber. In the aforementioned algorithms, the signal amplitude at the receiver and/or transmitter is assumed to be an accurate estimator of the signal profile in the fiber, and nonlinear compensation is applied using the received and/or transmitted signal(s). For zerodispersion fiber, in which signal amplitude changes are small, arising only from noise, this is a good assumption, so performance improvement has been observed in submarine systems. In systems where local dispersion exceeds a symbol period, however, nonlinear phase rotation fails (Ip \& Kahn, 2008), as interaction between intersymbol interference (ISI) and nonlinearity gives rise to "intra-channel" nonlinear effects, which have been studied in (Essiambre et al, 1999). As a first-order approximation, it has been assumed that the nonlinear phase distortion at a given symbol depends not only on the instantaneous amplitude, but on the amplitude profile of the received signal spanning a time interval equal to the fiber's impulse response duration. An intra-channel four-wave mixing (IFWM) compensation scheme was studied in (Lau et al., 2008), and can lead to a small improvement in performance.

The ultimate nonlinear compensation method is backpropagation, which inverts the NLSE. In this framework, receiver-based optical dispersion compensation or electronic linear equalization can be seen as special cases of backpropagation where the receiver inverts only chromatic dispersion (CD). Similarly, mid-span phase conjugation is an example of backpropagation where the second half of the transmission link inverts the impairments of the first half (Chowdhury \& Essiambre, 2004). Electronic backpropagation was first studied in (Roberts et al., 2006) as a transmitter-side compensation method, since in the absence of coherent detection, manipulation of the field is only possible at the modulator. With coherent detection, however, recovery of the received electric field enables receiver-side backpropagation, which was first studied in (Mateo et al, 2008). Receiver-side backpropagation has the advantage that it can ultimately be adaptive without the need for a feedback channel.

In this work, we review the principles of backpropagation and present recent results. Since backpropagation requires solving the NLSE, we begin by reviewing techniques for finding its numerical solutions. This is an area which has been well studied in simulation of optical networks. We will review split-step methods, emphasizing the length scales of different fiber effects, the computational costs of different algorithms, the step size requirements, and their dependence on system parameters. Backpropagation will then be introduced, firstly for single-polarization transmission for a single wavelength channel. In later sections, we will generalize backpropagation for wavelength-division-multiplexed (WDM) systems and for dual-polarization transmission. Simulation results will be presented.

\section{Signal Propagation}

\subsection{Nonlinear Schrödinger Equation (NLSE)}

Propagation in optical fiber is affected by a combination of linear and nonlinear phenomena. At low symbol rates and short transmission distances, where pulse spreading due to polarization-mode dispersion (PMD) is confined well within a symbol period, we can model signal propagation by a scalar nonlinear Schrödinger equation (NLSE) (Agrawal, 2001):

$$
\frac{\partial E}{\partial z}=(\hat{D}+\hat{N}) E
$$




$$
\begin{gathered}
\hat{D}=-\frac{1}{2} \alpha-j \frac{\beta_{2}}{2 !} \frac{\partial^{2}}{\partial t^{2}}+\frac{\beta_{3}}{3 !} \frac{\partial^{3}}{\partial t^{3}} \\
\hat{N}=j \gamma|E|^{2}
\end{gathered}
$$

where $E$ is the signal electric field, $\hat{D}$ and $\hat{N}$ are the linear and nonlinear operators, and $\alpha, \beta_{2}, \beta_{3}$ and $\gamma$ are the fiber's attenuation, group velocity dispersion, dispersion slope, and nonlinear coefficients, respectively. In (1)-(3), we have specified the electric field in a moving co-ordinate system that propagates at the group velocity of the fiber, so the $\partial / \partial t$ term has been neglected.

It has been shown that the NLSE is an accurate model for a wide variety of optical phenomena, including single-wavelength and dense wavelength-division-multiplexed (DWDM) systems, solitons, and ultra-short pulses down to a few optical cycles in duration. Only in very special conditions (e.g., solitons) does the NLSE yield analytical solutions. In general, the NLSE must be solved numerically. Various solution methods have been developed, including finite-difference methods and split-step methods (SSMs). The latter are generally preferred because of the computational advantage of the fast Fourier transform and related transforms.

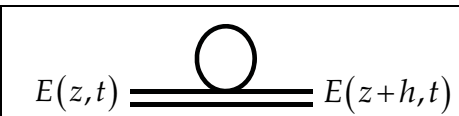

(a)

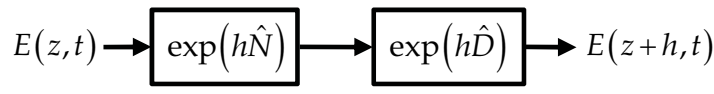

(b)

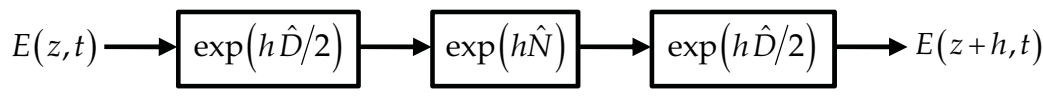

(c)

Fig. 1. (a) Propagation through a fiber section of length $h$ modeled using the (b) asymmetric split-step method, and (c) symmetric split-step method.

In the split-step method, the fiber is divided into sections, where for each section we perform the integration:

$$
E(z+h, t)=\exp (h(\hat{D}+\hat{N})) E(z, t) .
$$

Although the linear and nonlinear operators can each be evaluated exactly when they appear in isolation, this is not the case in (4). Two popular approximations to (4) are the asymmetric split-step method (A-SSM) approximation (Fig. 1a):

$$
E(z+h, t) \approx e^{h \hat{D}} e^{h \hat{N}} E(z, t),
$$

and the symmetric split-step method (S-SSM) approximation (Fig. 1b), where the linear operator is split into two parts to be evaluated on either side of the nonlinear operator:

$$
E(z+h, t) \approx e^{h \hat{D} / 2} e^{h \hat{N}} e^{h \hat{D} / 2} E(z, t) .
$$

The accuracy of the two approximations can be compared using the Baker-Hausdorff formula (Weiss \& Maradudin, 1962): 


$$
\exp (\hat{a}) \exp (\hat{b})=\exp \left(\hat{a}+\hat{b}+\frac{1}{2}[\hat{a}, \hat{b}]+\frac{1}{12}[\hat{a}-\hat{b},[\hat{a}, \hat{b}]]+\cdots\right),
$$

where $[\hat{a}, \hat{b}]=\hat{a} \hat{b}-\hat{b} \hat{a}$ is the commutator. It can be shown that the A-SSM and S-SSM lead to:

$$
\begin{gathered}
e^{h \hat{D}} e^{h \hat{N}}=\exp \left(h(\hat{D}+\hat{N})+\frac{1}{2} h^{2}[\hat{D}, \hat{N}]+\cdots\right) . \\
e^{h \hat{D} / 2} e^{h \hat{N}} e^{h \hat{D} / 2}=\exp \left(h(\hat{D}+\hat{N})+\frac{1}{6} h^{3}\left[\hat{N}+\frac{\hat{D}}{2},\left[\hat{N}, \frac{\hat{D}}{2}\right]\right]+\cdots\right) .
\end{gathered}
$$

In (8) and (9), all other terms inside the exponential apart from $h(\hat{D}+\hat{N})$ are error terms.

When high accuracy is required, the S-SSM has superior performance because the error is third order in $h$, whereas the error in the A-SSM is only second order in $h$. Split-step methods with accuracy higher than third order have been studied in (Muslu \& Erbay, 2005), but have higher complexity. In backpropagation, high accuracy is generally not required because the signal is already corrupted by noise. Depending on the SNR and the power penalty requirement, a global relative accuracy of $\sim 10^{-3}$ is typically sufficient. Thus, computational cost, and the ability to parallelize and pipeline the algorithm are more pertinent considerations for real-time implementations. The S-SSM is considered here because it is possible to combine the linear half-step operators between adjacent sections so there is no difference between the complexities of S-SSM and the A-SSM (Fig. 3c).

To propagate a signal using (5) or (6), we can evaluate the linear operator as a multiplication by an all-pass filter in the frequency domain:

$$
\begin{aligned}
\mathscr{F}\{\exp (h \hat{D}) E(z, t)\} & =\exp (h \mathscr{T}\{\hat{D}\}) \mathscr{T}\{E(z, t)\} \\
& =\exp \left(\left(-\frac{\alpha}{2}+j \frac{\beta_{2}}{2 !} \omega^{2}+j \frac{\beta_{3}}{3 !} \omega^{3}\right) h\right) E(z, \omega)
\end{aligned}
$$

where $E(z, \omega)$ is the Fourier transform of the time-domain signal $E(z, t)$. This is known as the split-step Fourier method (SSFM).

The nonlinear operator, on the other hand, is most easily evaluated as a phase rotation in time:

$$
\exp (h \hat{N}) E(z, t)=\exp \left(j \gamma h|E(z, t)|^{2}\right) E(z, t) .
$$

In contrast with the linear operator, the nonlinear operator depends on signal amplitude. One way to improve the accuracy of the SSM is to use the trapezoidal rule for the integral of $\hat{N}$. For the S-SSM, we have (Agrawal, 2001):

$$
\begin{aligned}
E(z+h, t) & =\exp \left(\frac{h}{2} \hat{D}\right) \exp \left(\int_{z}^{z+h} \hat{N}\left(z^{\prime}\right) d z^{\prime}\right) \exp \left(\frac{h}{2} \hat{D}\right) E(z, t) \\
& \approx \exp \left(\frac{h}{2} \hat{D}\right) \exp \left(h \frac{\hat{N}(z+h)+\hat{N}(z)}{2}\right) \exp \left(\frac{h}{2} \hat{D}\right) E(z, t) .
\end{aligned}
$$


Since $\hat{N}(z+h)=j \gamma|E(z+h, t)|^{2}$ depends on the output, (12) is solved iteratively using the numerical procedure in Fig. 2. We initially assume that $\hat{N}(z+h) \approx \hat{N}(z)$. Using (12), we compute an updated estimate of the output, $E_{\text {new }}(z+h, t)$, which enables a new estimate for $\hat{N}(z+h)$. We then apply (12) again, and so forth, until convergence or until a preset number of iterations is reached. This method is known as the iterative, symmetric SSFM (IS-SSFM). Since iteration is time-consuming, a faster, but less accurate non-iterative method can also be used. These are:

$$
\begin{gathered}
E(z+h, t) \approx \exp (h \hat{D}) \exp (h \hat{N}(z)) E(z, t), \text { and } \\
E(z+h, t) \approx \exp \left(\frac{h}{2} \hat{D}\right) \exp (h \hat{N}(z+h / 2)) \exp \left(\frac{h}{2} \hat{D}\right) E(z, t)
\end{gathered}
$$

for the A-SSM and S-SSM, respectively. Note that for non-iterative case (NA-SSM), the order of the operators is based on the heuristic that nonlinear effects occur at high power, which is typically found at the beginning of a fiber section unless backward Raman pumping is used, so for large step sizes, the nonlinear operator should be applied first. ${ }^{1}$

The SSFM algorithm is thus summarized in Fig. 3. The linear and nonlinear operators are successively evaluated in the frequency- and time-domains, with fast Fourier transforms (FFT) and inverse fast Fourier transforms (IFFT) used to switch between the domains. The complexity of the algorithm is dominated by the Fourier transforms, since the evaluation of an $N$-point FFT or IFFT requires $O(N \log N)$ operations, whereas the filtering and phase rotation operations in (10) and (11) are only $O(N)$.

\footnotetext{
${ }^{1}$ In backpropagation, we would apply the linear operator first.
} 


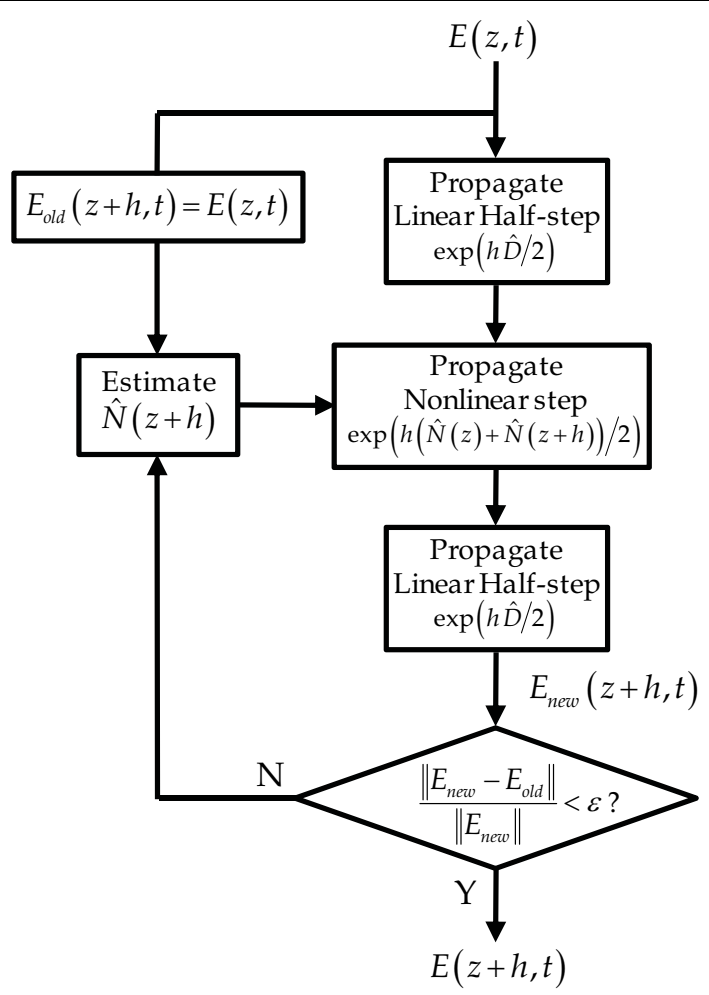

Fig. 2. Iterative, symmetric split-step method evaluation.

In offline simulations, it is possible to take the FFT/IFFT of the entire signal. This approach has high computational cost due to a large $N$, and cannot work in real-time systems. As (10) is a linear filtering operation, well-known methods can be used to design a digital filter that approximates $H(\omega)=\exp \left(\left(-\frac{\alpha}{2}+j \frac{\beta_{2}}{2 !} \omega^{2}+j \frac{\beta_{3}}{3 !} \omega^{3}\right) h\right)$ over the frequency range of interest, $|\omega| \leq 2 \pi / T$, where $T$ is the sampling period. If a finite-impulse response (FIR) filter is employed, the required filter length for a target accuracy will be proportional to the impulse duration of $\sim 2 \pi\left|\beta_{2}\right| / T^{2}$ samples. It is also possible to use an infinite-impulse response (IIR) filter to reduce the tap requirement (Plura et al., 2001). However, IIR filters are not desirable for real-time implementation because the delay of digital multipliers is typically much longer than a symbol period. Hence, an IIR filter output can only respond to input samples after this multiple-symbol delay has elapsed. Since FIR filters have no feedback, they are not constrained by delay. Moreover, FIR filters can be parallelized and efficiently implemented in the frequency-domain (Oppenheim \& Schafer), and are therefore more suitable for realtime implementation. Other methods of evaluating the linear operator, such as using wavelets have studied in (Kremp \& Freude, 2005; Goldfarb \& Li, 2009), and may lead to further computational savings compared to the standard SSFM. 


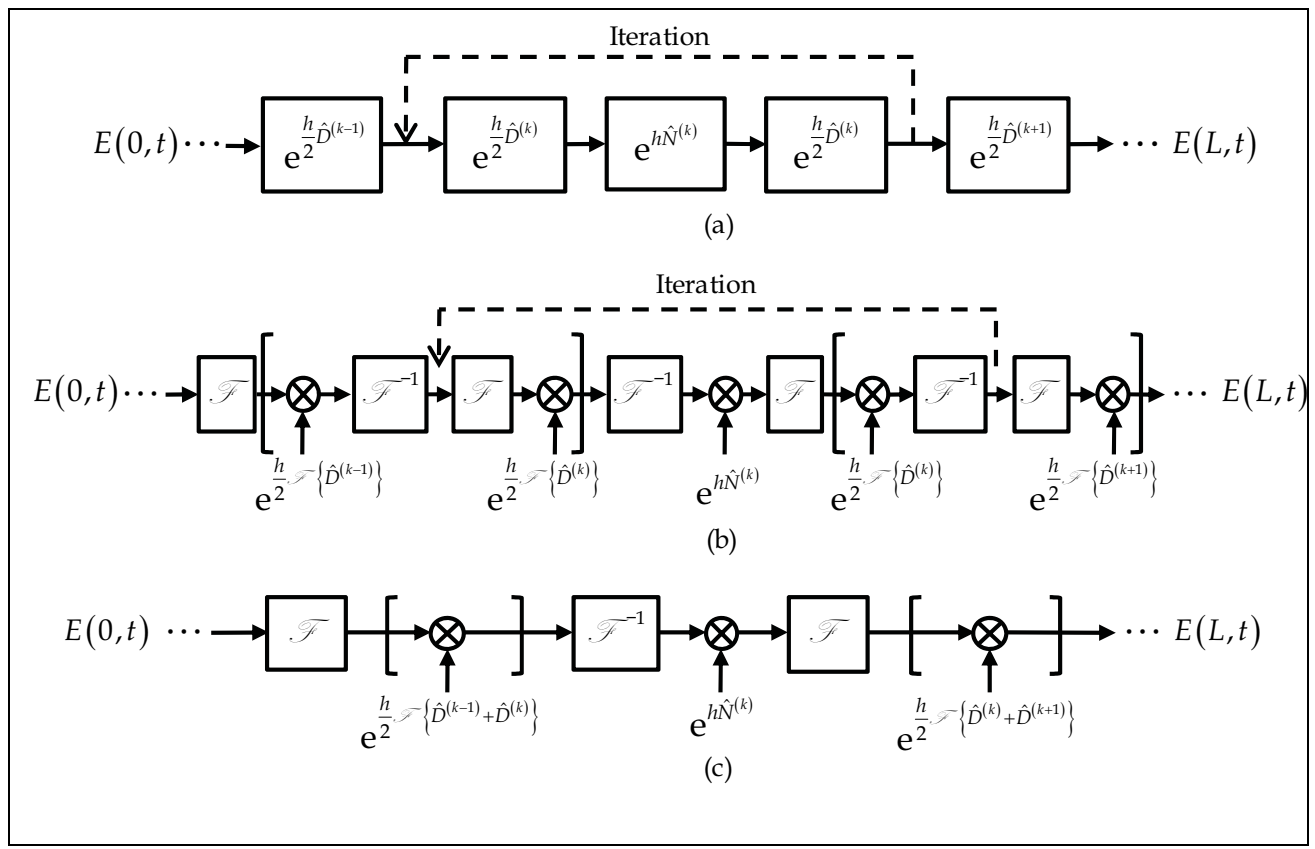

Fig. 3. (a) S-SSM with optional iterative procedure for solving each section. (b) S-SSFM with optional iterative procedure for solving each section. (c) If non-iterative method is used, adjacent linear operators can be combined so the total complexity is the same as the A-SSM.

\subsection{Step Size Selection}

The SSFM can be made increasingly accurate by reducing the step size $h$. For a given numerical accuracy, the step size requirement was studied in (Sinkin et al., 2003) and (Zhang \& Hayee, 2008). Traditionally, the step size was selected based on the characteristic lengths of the system, which are the distances over which each impairment induces one radian of phase distortion. For $\mathrm{CD}$ and nonlinearity, these are:

$$
\begin{gathered}
l_{C D} \sim 2 / \beta_{2} \omega_{\max }^{2}, \text { and } \\
l_{N L} \sim 1 / \gamma P_{t x},
\end{gathered}
$$

where $\omega_{\max }$ is the highest frequency component of the signal and $P_{t x}$ is the launched power. ${ }^{2}$ We can bound the maximum distortion of each step by setting:

$$
h=\min \left(\Phi_{C D} l_{C D}, \Phi_{N L} l_{N L}\right) .
$$

The choice of $\Phi_{C D}$ and $\Phi_{N L}$ depends on the accuracy required. Since CD is usually the larger impairment, (Sinkin et al., 2003) found that setting $h$ based on CD walkoff length

2 Strictly speaking, no signal is bounded in frequency. For single-carrier signals, we can define $\omega_{\max }=2 \pi R_{s}$ where $R_{s}$ is the symbol rate. For OFDM, we define $\omega_{\max }=2 \pi\left(N_{u}+1\right) \Delta f_{s}$, where $N_{u}$ is the number of used subcarriers and $\Delta f_{s}$ is the subcarrier frequency spacing. 
gives good numerical performance for both single- and multi-channel systems over the accuracy range of interest.

Another approach is to bound the error of each step directly. (Sinkin et al., 2003) proposed a "local error method" approach, where the step size is varied throughout a fiber to ensure that the relative local error $\delta=\left\|E_{t}-E_{S S M}\right\| /\left\|E_{t}\right\|$ between the numerical SSM solution and the true NLSE solution is constant for every step. Relative local error is related to the closeness with which the A-SSM and S-SSM in (5) and (6) approximate the integral in (4). According to (8) and (9), the error in the exponential involves the commutator of $\hat{D}$ and $\hat{N}$. (Rieznik et al., 2005) used quantum mechanics to derive results, while (Zhang \& Hayee, 2008) used a related approach that considered the change in pulse width when the ordering of operators is swapped. Both analyses produced the same result that to keep relative local error constant, the step sizes of the A-SSM and S-SSM should be:

$$
\begin{gathered}
h^{2}(z)=\Delta \varsigma /\left[\gamma P(z)\left|\beta_{2}\right| \omega_{\max }^{2}\right], \text { and } \\
h^{3}(z)=\Delta \xi /\left[\gamma P(z)\left(\left|\beta_{2}\right| \omega_{\max }^{2}\right)^{2}\right] .
\end{gathered}
$$

In comparison with (17), the dispersion and nonlinear parameters have a multiplicative contribution in $h$. This is intuitively satisfying, since the NLSE can be solved analytically in the limit when either dispersion or nonlinearity is zero, allowing the use of an arbitrarily large step size. Secondly, the step size requirement varies with $z$ because power is decreasing with distance.

Ultimately, signal propagation involves evaluating many steps of the SSM. The parameter of interest is not the error of each step, but the global relative error between the numerical solution and the true NLSE solution over the entire propagation path. In WDM transmission over multiple spans of fiber, (Zhang \& Hayee, 2008) showed that global error grows linearly with the number of spans. To keep global error constant, $\Delta \varsigma / h$ or $\Delta \xi / h$ should be kept constant. For the A-SSM, $h$ should therefore vary as $1 /\left|\beta_{2}\right|, 1 / \omega_{\max }^{2}$ and $1 / P(z)$ with respect to the dispersion strength, signal bandwidth, and signal power; while for the S-SSM, $h$ should vary as $1 /\left|\beta_{2}\right|, 1 / \omega_{\max }^{2}$ and $1 / \sqrt{P(z)}$. These rules provide useful guidelines on the change in the complexity of backpropagation as system parameters are varied. For example, a WDM signal with $N_{c h}$ channels has roughly $N_{c h}$ times the bandwidth and $N_{c h}$ the power of a single channel, so using the S-SSM, complexity increases as $N_{c h}^{5 / 2}$. Whereas a typical step size for simulating a single wavelength channel is tens of kilometers, the requirement for a 16-channel WDM signal is reduced to less than 100 meters.

Finally, although local error methods may be computationally efficient, a variable step size may not be desirable because it will require different filter coefficients and FFT sizes for each linear operator of the different sections. Practical implementation may dictate using a constant step size for each fiber type in the channel, in which case, (18) or (19) may still be used, but a single $h$ is computed for each fiber replacing $P(z)$ with the maximum power $P_{\max }$. 


\section{System Model}

We use the generalized transmission model shown in Fig. 4. At the transmitter, a digital signal processor (DSP) converts input symbols into four digital signals corresponding to the I and $\mathrm{Q}$ components of the two transmitted polarizations. These are converted to analog by arbitrary waveform generators represented by digital-to-analog converters (D/A), driving a pair of Mach-Zehnder (MZ) modulators that perform upconversion onto an optical carrier. The channel is comprised of a concatenation of single-mode fiber (SMF) spans, with inline amplification and dispersion-compensation fiber (DCF) after each span. At the receiver, the optical signal is mixed with a local oscillator $(\mathrm{LO})$ laser through a network of optical hybrids and photodetectors (PD), yielding electronic signals corresponding to the $\mathrm{I}$ and $\mathrm{Q}$ components of the two received polarizations. These are synchronously sampled and digitized by analog-to-digital (A/D) converters at a rate of $1 / T=(M / K)\left(1 / T_{s}\right)$, where $T_{s}$ is the symbol period and $M / K$ is a rational oversampling rate, and then processed by a DSP.

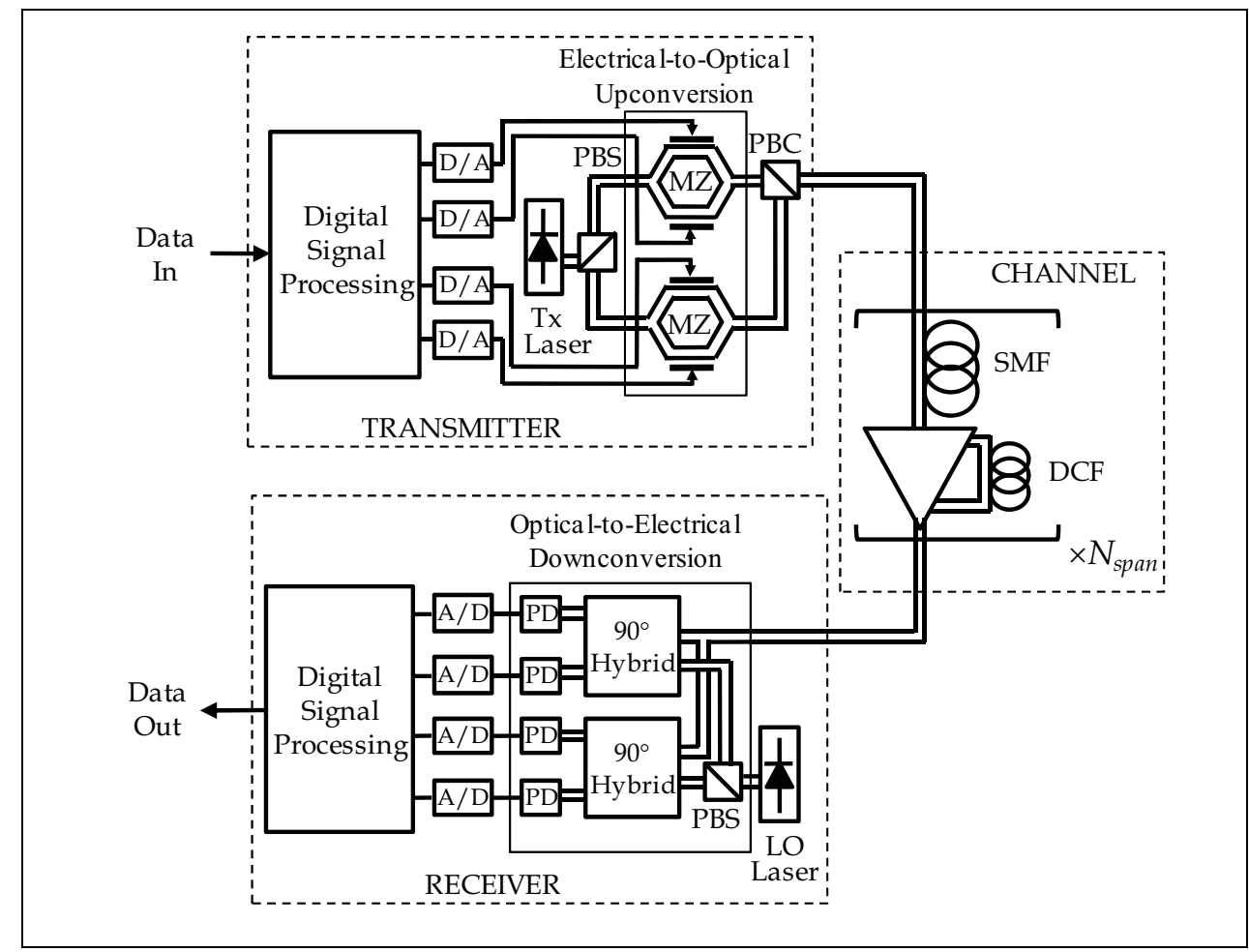

Fig. 4. Coherent system with digital signal processing at the receiver and/or transmitter.

A defining feature of the system shown is that baseband signals equivalent to the transmitted (and received) optical fields are available at the transmitter (and receiver). This enables an arbitrary waveform be transmitted over the channel, and enables arbitrary manipulation of the received waveform for recovery of the transmitted symbols. For 
example, the transmitter DSP can implement any signal pre-distortion algorithms, including $\mathrm{CD}$ pre-compensation, nonlinear phase noise pre-compensation or transmitter-side backpropagation. Similarly, the receiver DSP can implement any algorithm to combat signal distortion by the channel, including linear equalization, nonlinear phase noise postcompensation, receiver-side backpropagation, carrier recovery and clock recovery. We note that Fig. 4 is a canonical model for any modulation format. In OFDM, for example, the DSP algorithm at the transmitter and receiver include FFT and IFFT operations to convert between frequency-domain symbols and time-domain signals. For single-polarization transmission, the unneeded MZ modulator and D/As can be removed; while for singlecarrier transmission, pulse shaping can be performed digitally by the DSP, or a pair of MZ modulators for pulse carving can be inserted before or after the data modulators.

\section{Backpropagation}

The NLSE is an invertible equation. In the absence of noise, the transmitted signal can be exactly recovered by "backpropagating" the received signal through the inverse NLSE given by:

$$
\frac{\partial E}{\partial z}=(-\hat{D}-\hat{N}) E
$$

This operation is equivalent to passing the received signal through a fictitious fiber having opposite-signed parameters (Fig. 5a). It is also possible to perform backpropagation at the transmitter side by pre-distorting the signal to invert the channel, and then transmitting the pre-distorted waveform (Fig. 5b). In the absence of noise, both schemes are equivalent. We shall focus on receiver-side backpropagation.

Backpropagation operates directly on the complex-valued field $E(z, t)$. Hence, the technique is universal, as the transmitted signal can have any modulation format or pulse shape, including multicarrier transmission using OFDM. Moreover, as backpropagation simply involves finding a numerical solution to the NLSE, the methods and step size settings developed in Section 2 are applicable.

Some differences between optical system simulation and impairment compensation should be noted. In the former, knowing the input to a fiber enables the output be computed to arbitrary precision; whereas in backpropagation, noise prevents exact recovery of the transmitted signal. It has been demonstrated that in the presence of noise, a modified backpropagation equation is effective in compensating nonlinearity:

$$
E_{B P}(z, t)=\exp (-h(\hat{D}+\xi \hat{N})) E_{B P}(z+h, t),
$$

where $0 \leq \xi \leq 1$ is the fraction of the nonlinearity compensated. For every set of system parameters, there exists an optimum $\xi$ that minimizes the mean square error (MSE) between the transmitted signal $E(0, t)$ and the backpropagation solution $E_{B P}(0, t)$. In zerodispersion fiber, for example, where backpropagation is equivalent to nonlinear phase rotation, it was shown that $\xi=0.5$ is optimal (Ho \& Kahn, 2004).

The existence of an optimum $\xi$ can be appreciated by considering that in a typical fiber, the magnitude of the dispersion operator is much greater than the nonlinear operator. Thus, 
nonlinearity can be viewed as a perturbation to a mostly dispersive channel. The optimum phase to de-rotate at each backpropagation step depends on the accuracy of $E_{B P}(z, t)$ as an estimate of $E(z, t)$. The more accurately the receiver estimates $E(z, t)$, the closer we can set $\xi$ to one, since we are confident that the nonlinear phase rotation will lead to an an output closer to the original signal. Conversely, if $E(z, t)$ is not known accurately, error in amplitude will be converted to random phase rotations by the nonlinear operator $h \xi \hat{N}$, yielding an output that is even further away from the desired signal in Euclidean distance. Hence, the optimum $\xi$ depends on the received SNR as well as any uncompensated distortions that are present during backpropagation. In particular, simulation results in Section 4.1 will show that system performance depends on the dispersion map, owing to interaction between signal and noise. Thus, the optimum $\xi$ is also dependent on the dispersion map and other system parameters, and needs to be individually optimized for each application.

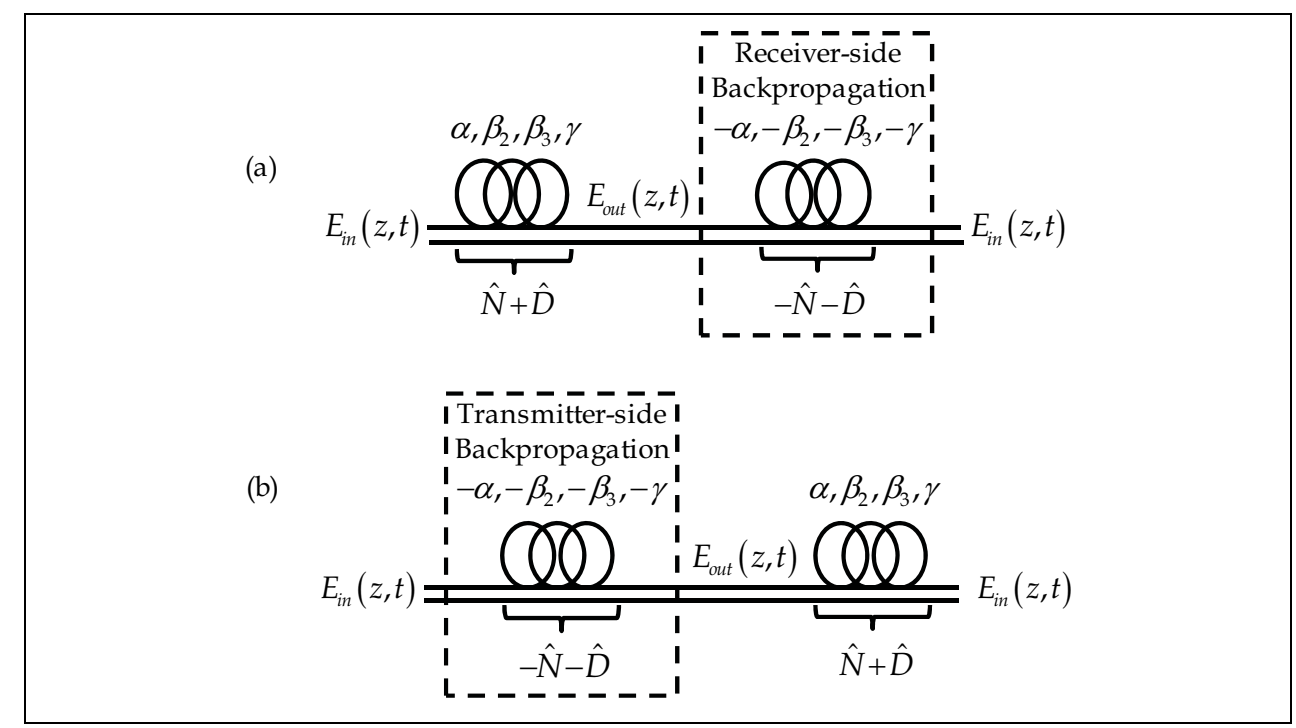

Fig. 5. (a) Transmitter-side and (b) receiver-side backpropagation.

\subsection{Single Channel Results}

The performance improvement enabled by backpropagation can be seen in Fig. 6, where $21.4 \mathrm{~Gb} / \mathrm{s}$ RZ-QPSK was transmitted over $25 \times 80-\mathrm{km}$ spans of SMF, with loss fully compensated after each span, and $10 \%$ residual dispersion compensation per span (Fig. 6a). The fiber and EDFA parameters are shown in Table 1, and the system dispersion map is shown in Fig. 6c. At intermediate points along the fiber, the accumulated dispersion (quantified in terms of group delay difference per unit wavelength) is much larger than a symbol interval, so the amplitude profile of the propagated signal differs significantly from that of either the transmitted or received signal. Interaction between dispersion and 
nonlinearity leads to strong IFWM, and the nonlinear phase de-rotation method (NLPN + Lin. Eq.) offers no performance gain over linear equalization alone (Fig. 6d). Fig. 7 shows the constellations obtained at the output of each method at a launched power of $0 \mathrm{dBm}$. Rather than spiral-shaped, circular clusters are observed in Fig. 7a, so nonlinear phase de-rotation is not effective. In contrast, even a crude backpropagation approximation: NA-SSFM with a step size equal to the length of a span (Fig. 6b), improves performance at high power (Fig. $6 d)$.

\begin{tabular}{|l||c|c||l|c|c|}
\hline & SMF & DCF & & $\begin{array}{c}\text { EDFA } \\
\# 1\end{array}$ & $\begin{array}{c}\text { EDFA } \\
\# 2\end{array}$ \\
\hline Attenuation $(\alpha)$ & $0.2 \mathrm{~dB} / \mathrm{km}$ & $0.6 \mathrm{~dB} / \mathrm{km}$ & Gain & $G$ & $G$ \\
\hline Dispersion $\left(\beta_{2}\right)$ & $17 \mathrm{ps} / \mathrm{nm}$ & $-80 \mathrm{ps} / \mathrm{nm}$ & Noise Figure & $5 \mathrm{~dB}$ & $5 \mathrm{~dB}$ \\
\hline $\begin{array}{l}\text { Nonlinear parameter } \\
(\gamma)\end{array}$ & $0.0013 \mathrm{~m}^{-1} \mathrm{~W}^{-1}$ & $0.0053 \mathrm{~m}^{-1} \mathrm{~W}^{-1}$ & & & \\
\hline Length $(L)$ & $80 \mathrm{~km}$ & $\begin{array}{c}0 \% \text { RDPS: } 17 \mathrm{~km} \\
10 \% \text { RDPS: } 15.3 \mathrm{~km} \\
100 \% \text { RDPS: } 0 \mathrm{~km}\end{array}$ & & & \\
\hline
\end{tabular}

Table 1. Fiber and EDFA simulation parameters.

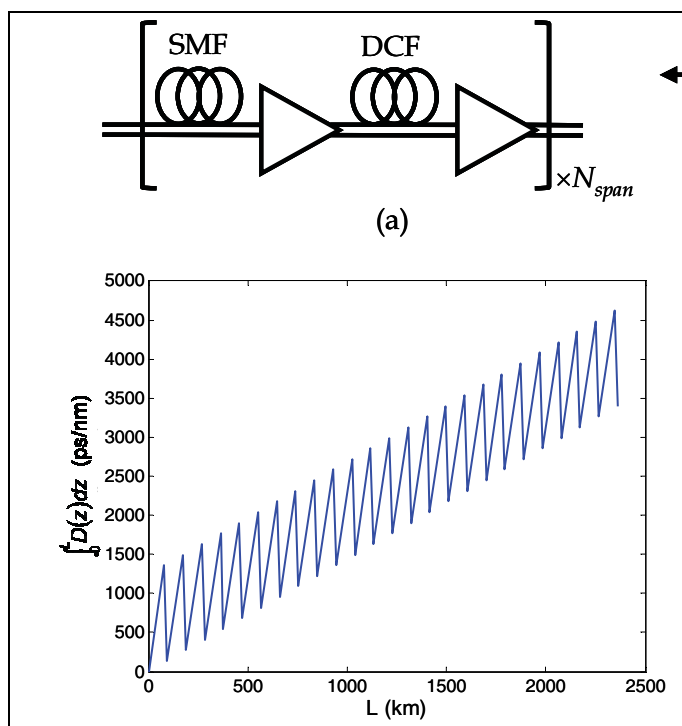

(c)

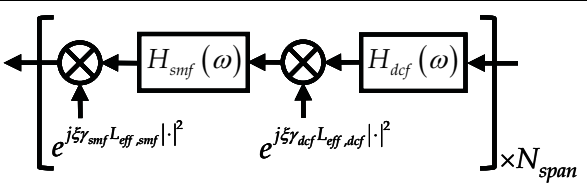

(b)

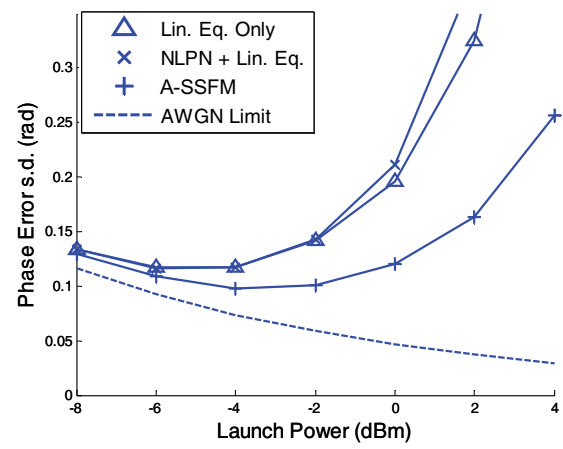

(d)

Fig. 6. (a) Long-haul transmission over multiple identical spans of fiber with amplification and dispersion compensation, (b) backpropagation model using A-SSFM with step size equal to a span length, (c) dispersion map, (d) system performance using different channel compensation algorithms for $21.4 \mathrm{~Gb} / \mathrm{s}$ RZ QPSK over $25 \times 80-\mathrm{km}$ SMF with $10 \%$ RDPS. 


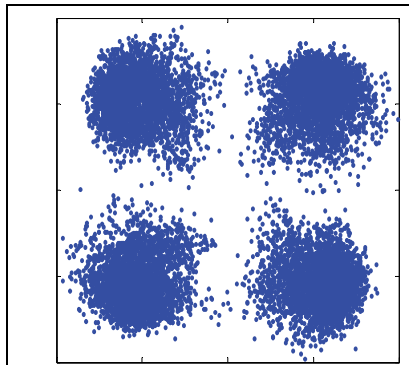

(a)

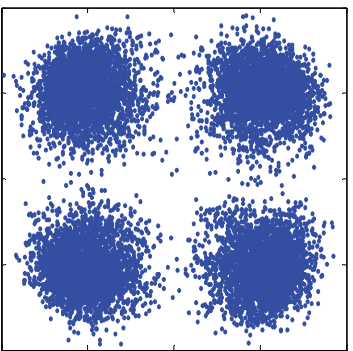

(b)

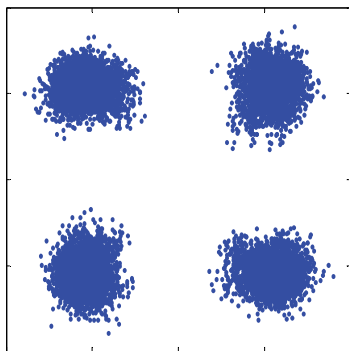

(c)

Fig. 7. Constellation diagrams obtained using (a) linear equalization only, (b) nonlinear phase noise compensation + linear equalization, and (c) backpropagation using A-SSFM with step size equal to a span length.

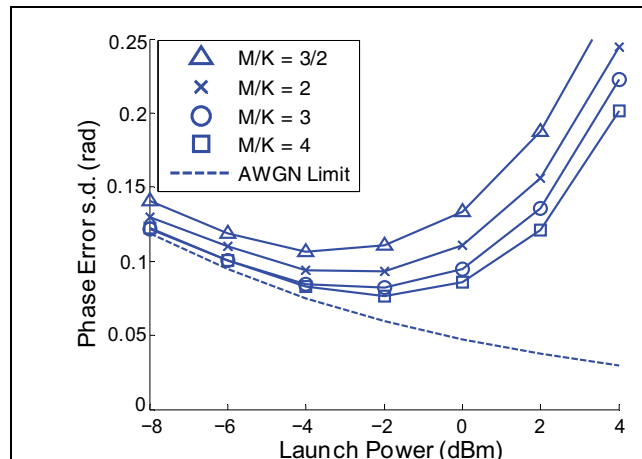

(a)

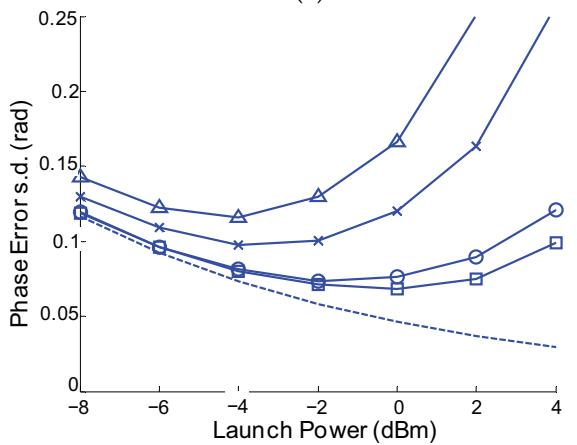

(c)

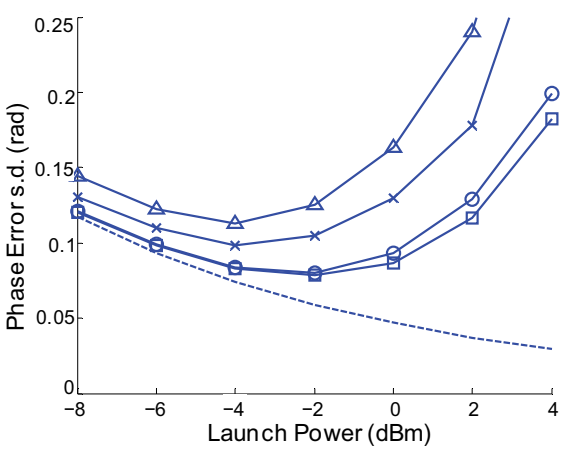

(b)

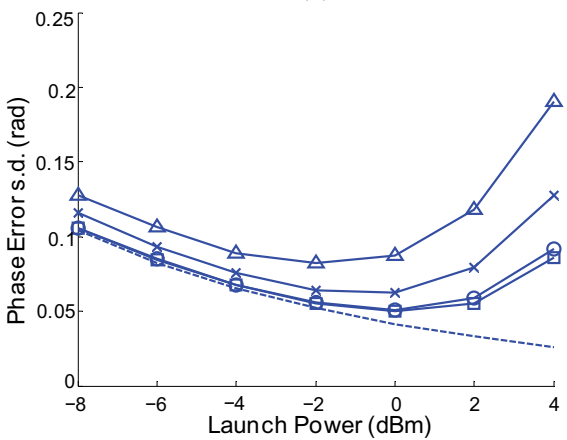

(d)

Fig. 8. System performance for $21.4 \mathrm{~Gb} / \mathrm{s}$ RZ QPSK over $25 \times 80-\mathrm{km}$ SMF with values of different residual dispersion per span (a) $0 \%$, (b) $5 \%$, (c) $10 \%$, and (d) $100 \%$.

System performance is strongly dependent on the dispersion map. In Fig. 8, RZ-QPSK at 21.4 Gb/s was again transmitted over $25 \times 80-\mathrm{km}$ spans of SMF with loss fully compensated after each span, but the residual dispersion per span (RDPS) was varied. In addition, 
different sampling rates were used for each map. It is observed that performance improves with (i) increased CD undercompensation, and (ii) increased oversampling. The first result can be explained because DCF incurs loss and nonlinearity. In coherent systems, CD can be compensated by a digital linear equalizer without undesired effects, so DCF is unnecessary and should be removed. This reduces the amplification requirement and thus optical amplifier noise. In addition, dispersion facilitates walkoff between the signal and out-ofband noise, reducing their nonlinear interactions. Increasing oversampling also improves performance because digital backpropagation becomes a better approximation to ideal analog backpropagation. As the nonlinear term in the NLSE is third order in the electric field, there is a performance gain when backpropagation is performed at an oversampling ratio of at least 3 .

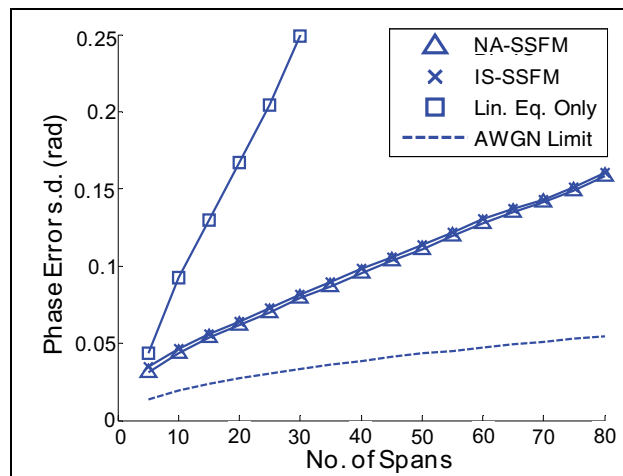

(a)

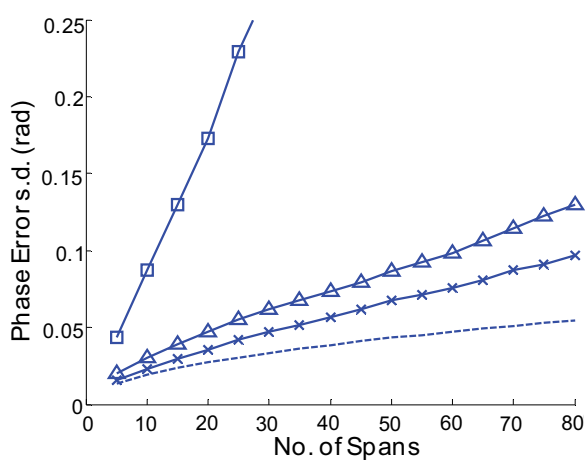

(b)

Fig. 9. System performance for $21.4 \mathrm{~Gb} / \mathrm{s}$ RZ QPSK $100 \%$ RDPS versus number of $80-\mathrm{km}$ spans at (a) $2 \times$ and (b) $3 \times$ oversampling.

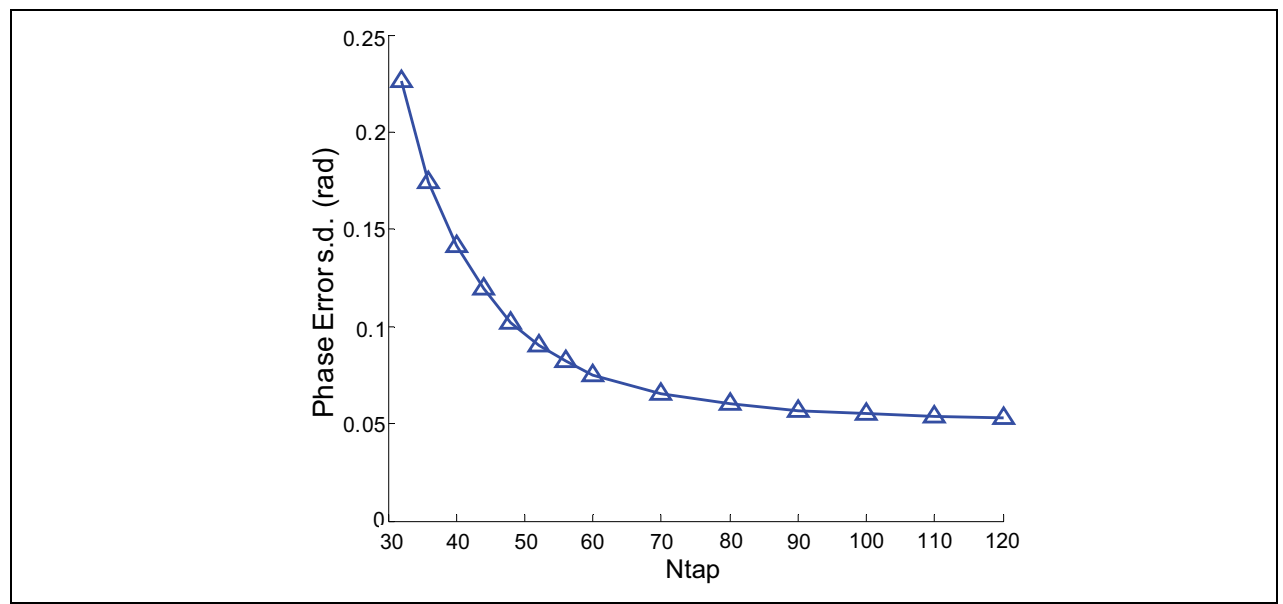

Fig. 10. Performance versus number of taps used to emulate linear operator for $21.4 \mathrm{~Gb} / \mathrm{s}$ RZ QPSK over $25 \times 80-\mathrm{km}$ SMF with $100 \%$ RDPS. 
With DCF removed, Fig. 9 shows the increase in transmission distance enabled by backpropagation. For a target bit-error ratio (BER) of $10^{-3}$, the phase error standard deviation should be less than 0.23 radians. Whereas linear equalization achieves a system reach of $2,000 \mathrm{~km}(25 \times 80-\mathrm{km}$ spans $)$, backpropagation extends this to over $6,400 \mathrm{~km}(80 \times 80$ $\mathrm{km}$ spans). At $2 \times$ oversampling, backpropagation using the IS-SSFM with 20 steps per span gives the same performance as the crude NA-SSFM method with span-length step size. This is because at a low oversampling rates, the backpropagated signal is not very accurate, and numerical errors due to insufficient sampling rate are the dominant effect. At $3 \times$ oversampling, IS-SSFM offers somewhat better performance, but has much higher computational cost.

To estimate the complexity of backpropagation, Fig. 10 shows the filter length required to emulate the linear operator in NA-SSFM for the system considered in Fig. 9, when $h$ is equal to one span at $3 \times$ oversampling. Approximately 70 taps are required. The most efficient realization of an FIR filter this length is frequency-domain implementation (Oppenheim \& Schafer), whose complexity is $\min _{B: N_{h}+B-1=2^{v}}\left(\frac{4}{B}\left(N_{h}+B-1\right)\left(\log _{2}\left(N_{h}+B-1\right)+1\right)\right)$ real multiplications per sample, where $B$ is the block size, and $N_{h}$ is the filter length. The nonlinear operator further requires 7 real multiplications per sample.

Table 2 compares the complexity of backpropagation with linear equalization, where the filter length requirement is $2 \pi\left|\beta_{2}\right| N_{\text {span }} L_{\text {span }} R_{s}^{2}(M / K)=47$ taps at $3 / 2 \times$ oversampling. The complexities of direct implementation of both methods are also shown. It is observed that backpropagation is two orders of magnitude more computationally expensive than linear equalization. We also note that the length of the linear operator, at 70 taps, is much larger than expected from $2 \pi\left|\beta_{2}\right| L_{\text {span }} R_{s}^{2}(M / K)$ (Ip, et al. 2008). This is due to amplitude ringing in the frequency domain when an FIR filter is designed using the windowing method. Since backpropagation requires multiple iterations of the linear filter, amplitude distortion due to ringing accumulates (Goldfarb \& Li, 2009).

\begin{tabular}{|c||c|c|}
\hline & Linear Equalization & Backpropagation \\
\hline Direct Implementation & 188 & 21515 \\
\hline $\begin{array}{c}\text { Frequency domain } \\
\text { implementation }\end{array}$ & 44 & 3992 \\
\hline
\end{tabular}

Table 2. System complexity in real multiplications per symbol for linear equalization and backpropagation for $21.4 \mathrm{~Gb} / \mathrm{s}$ RZ QPSK over $25 \times 80-\mathrm{km}$ SMF with $100 \%$ RDPS

\section{Multichannel Backpropagation}

In DWDM transmission, the ability to overcome interchannel nonlinear impairments depends on the system design. Consider the two systems shown in Fig. 11. In point-to-point transmission, cross-phase modulation (XPM) and four-wave mixing (FWM) are deterministic effects. Hence, they can be effectively compensated by backpropagation. In a mesh network, however, signals of different wavelengths can be dynamically routed by reconfigurable add/drop multiplexers (ROADMs). Signals received in adjacent channels may not be the same signals that were transmitted in adjacent channels. Two sources of 
error can arise when backpropagation is performed. Firstly, the receiver has no knowledge of the channels that were dropped, so nonlinear effects involving their fields cannot be compensated. Secondly, nonlinear effects arising from channels added at a intermediate nodes do not extend all the way back to the transmitter, hence when the received signal is fully backpropagated, spurious nonlinear effects will result. In the worst case, channels are either added or dropped just prior to the receiver ${ }^{3}$. Both uncompensated and spurious nonlinear effects can severely degrade system performance, especially if the channels added or dropped are near neighbors of the desired channel. Hence, backpropagation may not always be effective in mitigating nonlinear effects in mesh networks. In such systems, crosschannel nonlinearity may still be reduced using dispersion-unmanaged transmission, exploiting walkoff to suppress interchannel nonlinearity. The receiver can then perform single-channel backpropagation to mitigate SPM.

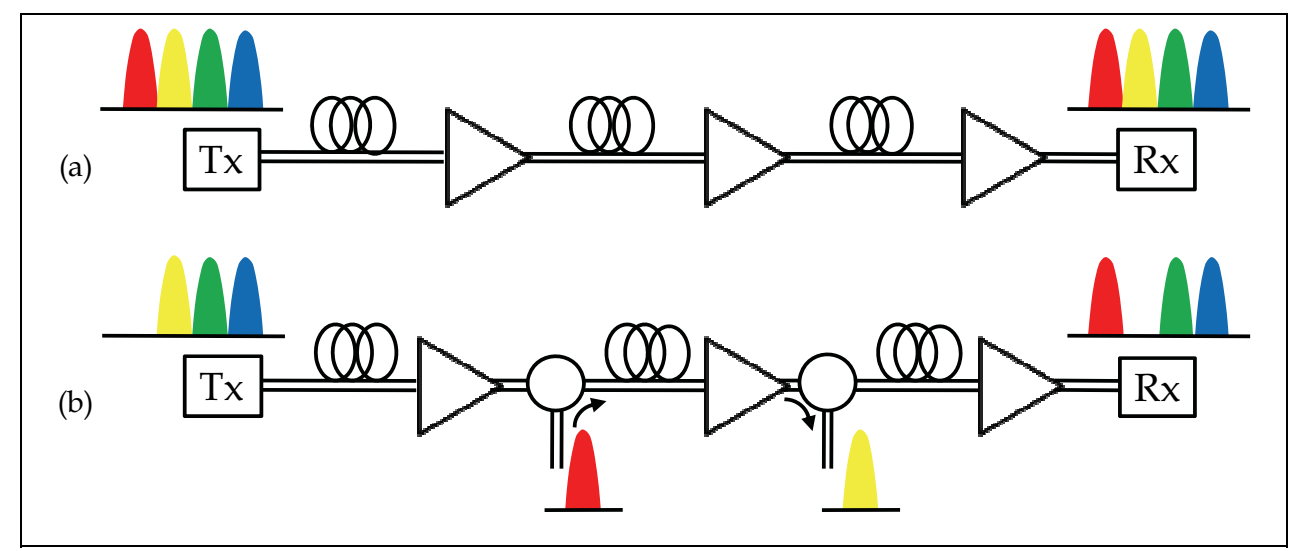

Fig. 11. Wavelength-division-multiplexed (WDM) transmission. (a) Point-to-point link, backpropagation can effectively mitigate interchannel nonlinearity, (b) Mesh network, backpropagation has limited benefit.

For point-to-point links, multichannel backpropagation can be used to mitigate interchannel nonlinearity. This was studied in (Mateo et al., 2008). Since the bandwidth of a DWDM signal is typically much greater than that of a single photoreceiver, we can need to use a structure like that shown in Fig 12a, where a bank of local oscillator lasers are tuned to the frequencies of the channels, translating the signal around each LO frequency to baseband. Provided the frequencies and phases of the local oscillators are synchronized, possibly using mode-locked lasers, the full electric field of the WDM signal can be reconstructed via the coherent sum:

$$
E(z, t)=\sum_{m} E_{m}(z, t) \exp (-j m \Delta \omega t),
$$

where $\Delta \omega$ is the channel spacing. Recovery of $E(z, t)$ enables the receiver to perform a "total-field" backpropagation using the procedure discussed in Section 2. The step size

\footnotetext{
${ }^{3}$ Conversely, for transmit-side backpropagation, the worst-case scenario involves channels added or dropped near the transmitter.
} 
equations derived previously can also be re-used by substituting $\omega_{\max }$ to be the bandwidth of the WDM signal, and $P_{t x}$ to be the total transmitted power.

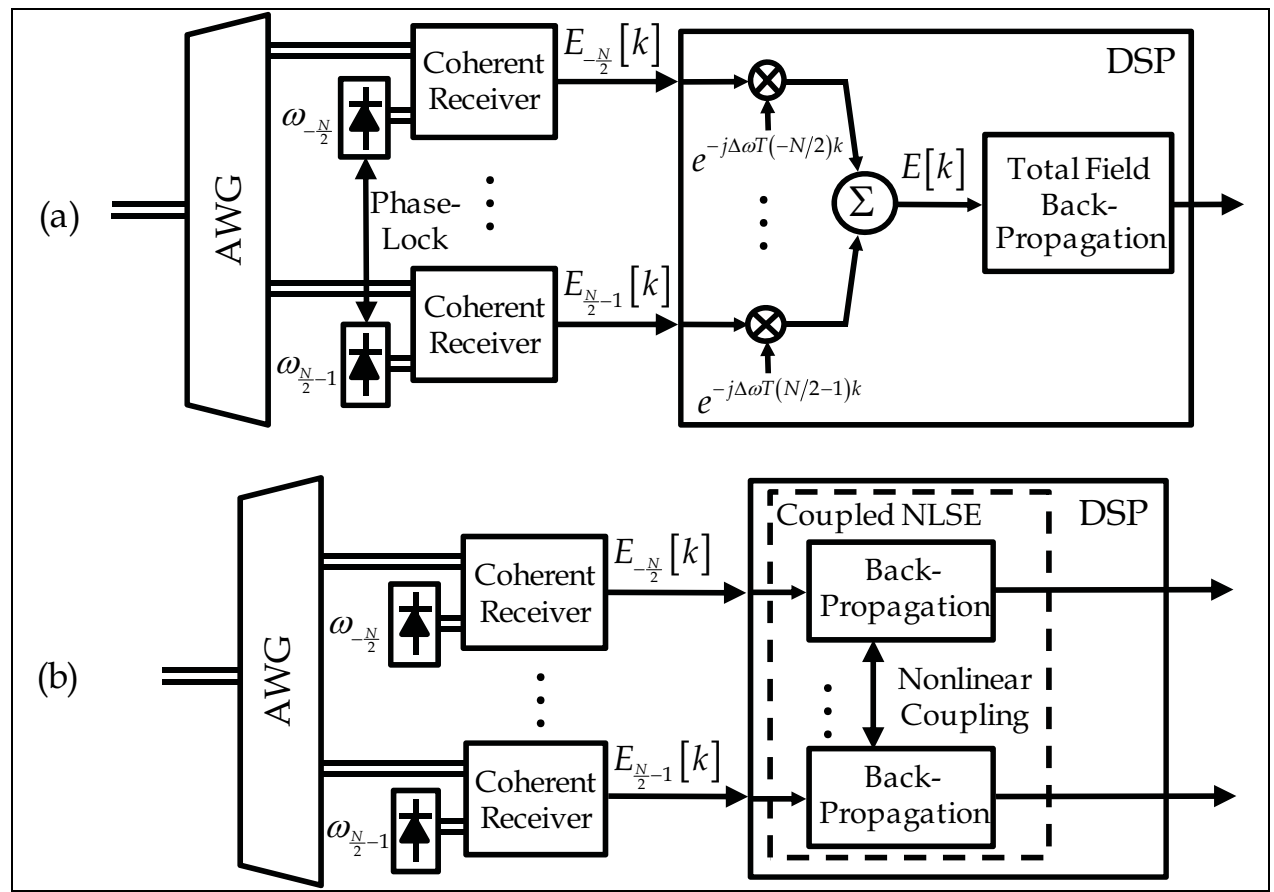

Fig. 12. Multichannel backpropagation using (a) total-field NLSE, (b) coupled NLSE.

In most installed fibers, CD results in low FWM efficiency. If (22) is substituted into the NLSE in (3), and the FWM terms are neglected, the total-field NLSE reduces to a set of coupled equations each describing the evolution of an individual WDM channel through the channel (Leibrich \& Rosenkranz, 2003):

$$
\begin{gathered}
\frac{\partial E_{i}}{\partial z}=\left(\hat{D}_{i}+\hat{N}_{i}\right) E_{i}, \\
\hat{D}_{i}=-\frac{1}{2} \alpha-\beta_{1, i} \frac{\partial}{\partial t}-j \frac{\beta_{2, i}}{2 !} \frac{\partial^{2}}{\partial t^{2}}+\frac{\beta_{3, i}}{3 !} \frac{\partial^{3}}{\partial t^{3}}, \\
\hat{N}_{i}=j \gamma\left(\left|E_{i}\right|^{2}+2 \sum_{j \neq i}\left|E_{j}\right|^{2}\right) .
\end{gathered}
$$

We observe that different DWDM channels have different group velocities, dispersions, and dispersion slopes, whose values are given by the Taylor Series expansion $\beta_{m, i}=\sum_{s} \beta_{m+s}(i \Delta \omega)^{s} / s !$, where $\beta_{k} \triangleq \beta_{0, k}$ denotes the values defined at the center wavelength. In addition, the nonlinear operator for channel-i in (25) has contributions from the fields of the other channels. But since this interaction only depends on the amplitude $\left|E_{j}\right|^{2}$ and is 
therefore phase-insensitive, synchronization of the local oscillators is not required. The coupled NLSE may therefore be more practical to implement than the total-field NLSE. The receiver structure for the coupled NLSE is shown in Fig. 12b.

\section{Dual-polarization Systems}

\subsection{Signal Propagation}

In real fibers, birefringence causes PMD. Since it is impractical - if not impossible - to estimate the Jones matrix of each increment in the fiber, the performance of backpropagation will be degraded. PMD may ultimately limit the signal bandwidth over which backpropagation can be performed. Additionally, polarization-multiplexed transmission enables the doubling of capacity keeping the same receiver sensitivity in SNR per bit. To study signal propagation in two polarizations, we use the vector NLSE:

$$
\begin{gathered}
\frac{\partial \mathbf{E}}{\partial z}=(\hat{\mathbf{D}}+\hat{\mathbf{N}}) \mathbf{E}, \\
\hat{\mathbf{D}}=-\frac{1}{2} \mathbf{\alpha}-\boldsymbol{\beta}_{1} \frac{\partial}{\partial t}-j \frac{1}{2 !} \boldsymbol{\beta}_{2} \frac{\partial^{2}}{\partial t^{2}}+\frac{1}{3 !} \boldsymbol{\beta}_{3} \frac{\partial^{3}}{\partial t^{3}}, \\
\hat{\mathbf{N}}=j \gamma\left[|\mathbf{E}|^{2} \mathbf{I}-\frac{1}{3}\left(\mathbf{E}^{H} \boldsymbol{\sigma}_{3} \mathbf{E}\right) \boldsymbol{\sigma}_{3}\right] .
\end{gathered}
$$

In these equations, $\mathbf{E}=\left[\begin{array}{ll}E_{x} & E_{y}\end{array}\right]^{T}$ is the Jones vector of the electric field, and $\boldsymbol{\alpha}, \boldsymbol{\beta}_{1}, \boldsymbol{\beta}_{2}$ and $\boldsymbol{\beta}_{3}$ are the loss, group velocity, $\mathrm{CD}$ and dispersion slope parameters. The $2 \times 2$ matrix representation of the parameters allows the most general polarization dependence. For example, setting a to have unequal eigenvalues enables polarization-dependent loss (PDL) be modeled. In comparison with (3), the nonlinear operator has an extra term that arises as a result of the cross-polarization nonlinearity. The matrix $\sigma_{3}$ is a Pauli spin matrix expressed in the standard notation of (Gordon \& Kogelnik, 2000):

$$
\sigma_{1}=\left[\begin{array}{cc}
1 & 0 \\
0 & -1
\end{array}\right], \sigma_{2}=\left[\begin{array}{ll}
0 & 1 \\
1 & 0
\end{array}\right] \text { and } \sigma_{3}=\left[\begin{array}{cc}
0 & -j \\
j & 0
\end{array}\right] \text {. }
$$

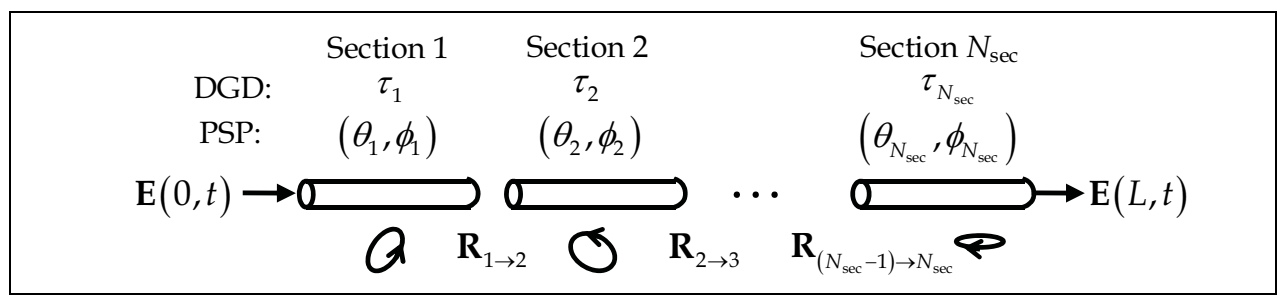

Fig. 13. Model of a fiber with polarization-mode dispersion.

In most fibers, the polarization dependence of $\boldsymbol{\alpha}, \boldsymbol{\beta}_{2}$ and $\boldsymbol{\beta}_{3}$ is negligible, so they may be replaced by scalars. To model arbitrary PMD of all orders, a fiber is typically divided into short sections, each possessing only first-order PMD, and the principal states of polarization 
(PSP) are randomly rotated between adjacent sections (Fig. 13). Thus, for the $k$-th fiber section, $\boldsymbol{\beta}_{1}$ can be expressed as:

$$
\boldsymbol{\beta}_{1}(z)=\mathbf{R}\left(\theta_{k}, \phi_{k}\right)\left(\frac{\delta}{2} \mathbf{\sigma}_{1}\right) \mathbf{R}^{H}\left(\theta_{k}, \phi_{k}\right),
$$

where,

$$
\mathbf{R}\left(\theta_{k}, \phi_{k}\right)=\left[\begin{array}{cc}
\cos \theta_{k} \cos \phi_{k}-j \sin \theta_{k} \sin \phi_{k} & \sin \theta_{k} \cos \phi_{k}+j \cos \theta_{k} \sin \phi_{k} \\
\sin \theta_{k} \cos \phi_{k}+j \cos \theta_{k} \sin \phi_{k} & \cos \theta_{k} \cos \phi_{k}+j \sin \theta_{k} \sin \phi_{k}
\end{array}\right]
$$

is a generalized polarization rotation matrix whose columns are the Jones vectors of the PSPs. The first column denotes the slow axis and is a unit vector on the Poincare sphere with azimuth and ellipticity angles of $2 \theta_{k}$ and $2 \phi_{k}$, respectively. $\delta_{k}$ is the differential group delay (DGD) of the section, and is a Maxwellian-distributed variable with mean $\bar{\tau}_{p m d} \sqrt{h}$, where $\bar{\tau}_{p m d}$ is the mean PMD of the fiber and $h$ is the section length.

In order to model PMD accurately, the section length should be less than the PMD characteristic length given by:

$$
l_{P M D} \sim\left(T_{s} / \bar{\tau}_{P M D}\right)^{2} \text {. }
$$

Typically, this number is large compared to the characteristic length of CD. Hence in practical fibers, the step sizes derived in Section 2.2 are sufficiently fine for accurate modeling of PMD.

The dual-polarization NLSE can be solved using the split-step methods introduced in Section 2 with the linear and nonlinear operators applied successively in the frequency- and time-domains. Note that for clarity, we have expressed (26) to (28) in Jones co-ordinates. For implementation, the nonlinear operator of the vector NLSE has a slightly simpler form when the electric field is specified in circular polarization co-ordinates.

\subsection{Backpropagation}

Like the scalar NLSE, the vector NLSE is invertible. In the absence of noise, knowledge of the fiber parameters enables backpropagation to exactly invert the channel. We define the backpropagation equation as:

$$
\mathbf{E}(z, t)=\exp (-h(\hat{\mathbf{D}}+\xi \hat{\mathbf{N}})) \mathbf{E}(z+h, t),
$$

where $\hat{\mathbf{D}}$ and $\hat{\mathbf{N}}$ are given in (27) and (28), and $0 \leq \xi \leq 1$ is a parameter to be optimized. We note that reversing the sign of the PMD term $-\boldsymbol{\beta}_{1}^{(k)}=\mathbf{R}\left(\theta_{k}, \phi_{k}\right)\left(-\frac{\delta}{2} \mathbf{\sigma}_{1}\right) \mathbf{R}^{H}\left(\theta_{k}, \phi_{k}\right)$ has the intuitive meaning of rotating the signal into the fiber PSPs and then undoing the section's DGD. The difference between the scalar and vector NLSE is that PMD makes the linear operator $h \hat{\mathbf{D}}(z)$ dependent on $z$ even if a constant step size is used. Fig. 14 shows dualpolarization backpropagation. The order of the linear operators applied should be in reverse order to forward propagation. 


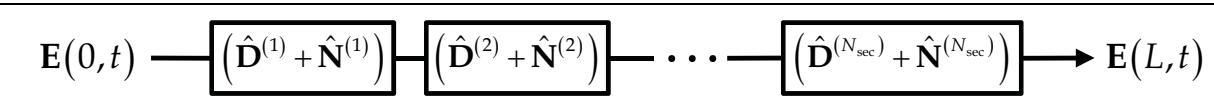

$$
\begin{aligned}
& \text { Fiber Section \#1 Fiber Section \#2 } \\
& \text { Fiber Section } \# N_{\text {sec }}
\end{aligned}
$$

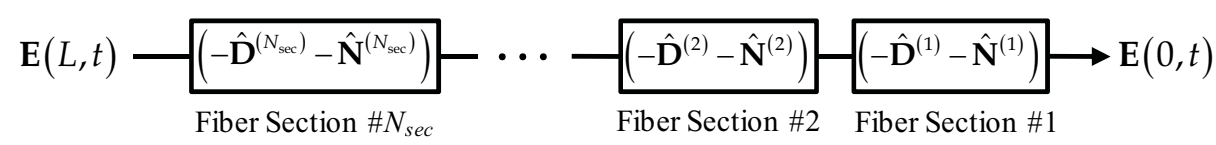

Fig. 14. Dual-polarization backpropagation.

If the Jones vector for each fiber section not be known at the receiver, the best estimate of the linear operator is:

$$
\hat{\mathbf{D}}^{n o p m d}=-\frac{1}{2} \boldsymbol{\alpha}-j \frac{1}{2 !} \boldsymbol{\beta}_{2} \frac{\partial^{2}}{\partial t^{2}}+\frac{1}{3 !} \boldsymbol{\beta}_{3} \frac{\partial^{3}}{\partial t^{3}} .
$$

The receiver shown in Fig. 15 can be used, where a linear equalizer follows backpropagation. In the absence of nonlinearity, backpropagation inverts the fiber $\mathrm{CD}$, so PMD is mitigated by the linear equalizer. At realistic transmission distances and symbol rates, PMD has only short duration, so we expect the signal amplitude profile will not be significantly distorted by PMD. Hence, backpropagation with the linear operator in (34) can still compensate most of the interactions between $\mathrm{CD}$ and nonlinearity. The linear equalizer compensates PMD and any residual linear effects not already compensated by backpropagation. This receiver structure is general. If backpropagation includes PMD, the linear equalizer is reduced to a fixed downsampler. 


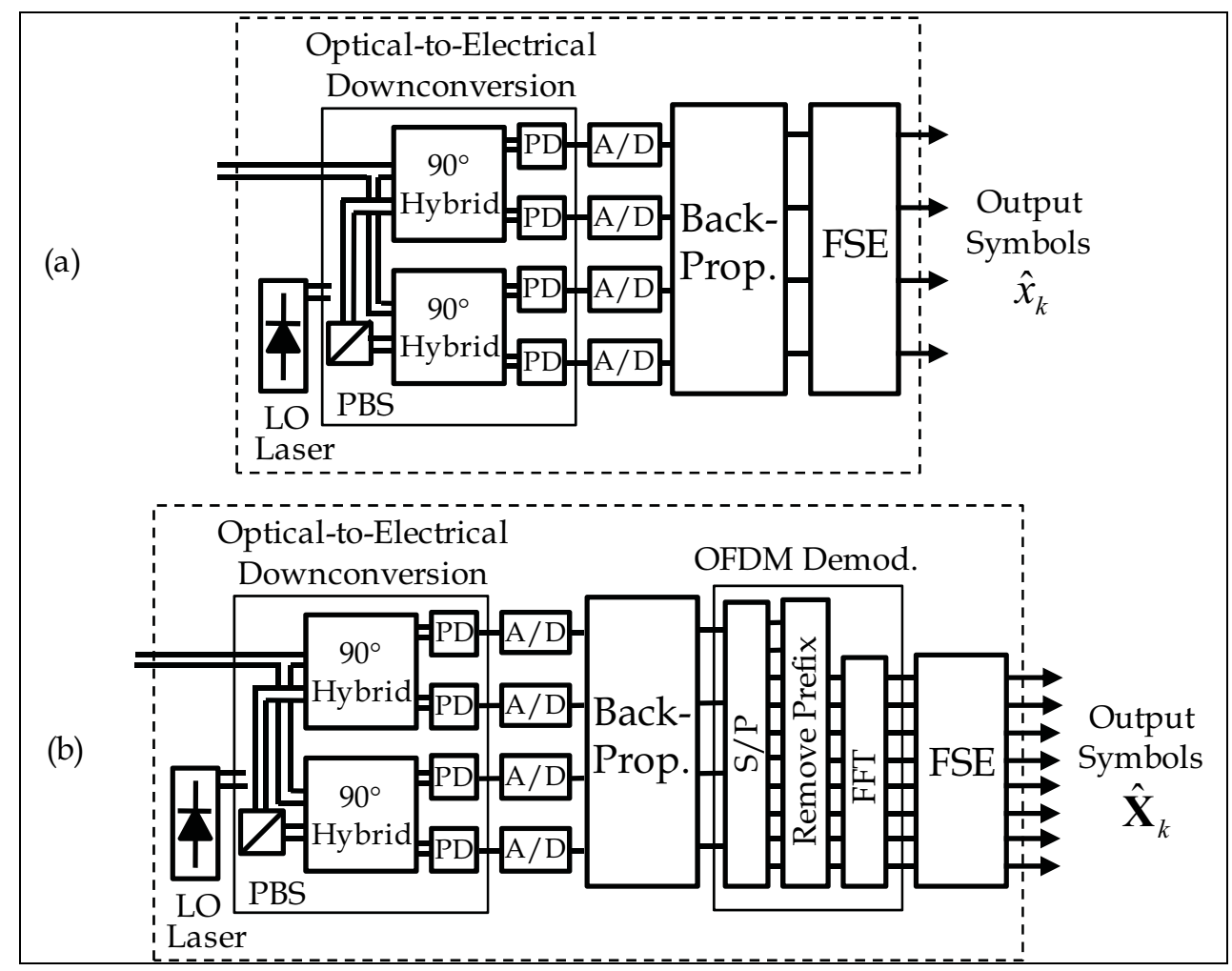

Fig. 15. Backpropagation receiver with output linear equalizer to mitigate residual linear distortion for (a) single-carrier, (b) OFDM.

\subsection{Results}

The performances of single-carrier and OFDM at $107 \mathrm{~Gb} / \mathrm{s}$ over $25 \times 80-\mathrm{km}$ SMF are compared for different dispersion maps in Fig. 16. The fiber and EDFA parameters are shown in Table 3. Gain is fully compensated after each span, but unlike Section 4, the individual gains of the two EDFAs $G_{1}$ and $G_{2}$ are optimized for best performance. For single-carrier, we assume polarization-multiplexed QPSK (PM-QPSK) with 50\% RZ pulse shaping, corresponding to 4 bits encoded per symbol. For OFDM, the FFT-size $\left(N_{c}\right)$, number of used subcarriers $\left(N_{u}\right)$ and prefix length $\left(N_{\text {pre }}\right)$ are shown for different dispersion maps in Table 4, and PM-QPSK was transmitted on all the used subcarriers. The metric of comparison is the phase error standard deviation, which in the absence of nonlinearity, satisfies $\sigma_{\varepsilon}=\sqrt{1 / 2 \gamma_{s}}$, where $\gamma_{s}$ is the SNR per symbol. For OFDM, all the subcarriers were found to have similar $\sigma_{\varepsilon}$, so the average $\sigma_{\varepsilon}$ across all the subcarriers is shown. In each plot shown in Fig. 16, linear equalization is compared to different backpropagation methods. ISSSFM denotes using the iterative, symmetric SSFM for solving the inverse NLSE, with the same step size equal to that used in simulating forward propagation. NA-SSFM denotes 
using the crude non-iterative, asymmetric SSFM with a step size equal to one span. For each method, comparison is made with the receiver including PMD ( $\hat{\mathbf{D}}$ given by (27)) and excluding PMD ( $\hat{\mathbf{D}}$ given by (34)) during backpropagation.

\begin{tabular}{|l||c|c||c|c|c|}
\hline & SMF & DCF & & $\begin{array}{c}\text { EDFA } \\
\# 1\end{array}$ & $\begin{array}{c}\text { EDFA } \\
\# 2\end{array}$ \\
\hline Attenuation $(\alpha)$ & $0.2 \mathrm{~dB} / \mathrm{km}$ & $0.5 \mathrm{~dB} / \mathrm{km}$ & Gain & $G_{1}$ & $G_{2}$ \\
\hline Dispersion $\left(\beta_{2}\right)$ & $17 \mathrm{ps} / \mathrm{nm}$ & $-85 \mathrm{ps} / \mathrm{nm}$ & Noise Figure & $4 \mathrm{~dB}$ & $4 \mathrm{~dB}$ \\
\hline $\begin{array}{l}\text { Dispersion Slope } \\
\left(\beta_{3}\right)\end{array}$ & $0.09 \mathrm{ps} / \mathrm{nm}^{2} / \mathrm{km}$ & $-0.3 \mathrm{ps} / \mathrm{nm}^{2} / \mathrm{km}$ & & & \\
\hline $\begin{array}{l}\text { Nonlinear parameter } \\
(\gamma)\end{array}$ & $0.0013 \mathrm{~m}^{-1} \mathrm{~W}^{-1}$ & $0.0053 \mathrm{~m}^{-1} \mathrm{~W}^{-1}$ & & & \\
\hline Mean PMD $\left(\bar{\tau}_{p m d}\right)$ & $0.1 \mathrm{ps} / \sqrt{\mathrm{km}}$ & $\begin{array}{c}0.1 \mathrm{ps} / \sqrt{\mathrm{km}} \\
0 \% \text { RDPS: } 16 \mathrm{~km} \\
10 \% \text { RDPS: } 14.4 \mathrm{~km} \\
100 \% \text { RDPS: } 0 \mathrm{~km}\end{array}$ & & & \\
\hline Length $(L)$ & $80 \mathrm{~km}$ & & & \\
\hline
\end{tabular}

Table 3. Fiber and EDFA simulation parameters.

\begin{tabular}{|l||c|c|c|}
\hline & $N_{\text {pre }}$ & $N_{u}$ & $N_{c}$ \\
\hline $0 \%$ RDPS & 4 & 13 & 16 \\
\hline $10 \%$ RDPS & 43 & 213 & 256 \\
\hline $100 \%$ RDPS & 391 & 1706 & 2048 \\
\hline
\end{tabular}

Table 4. OFDM parameters used for different dispersion maps.

The results show that at $0 \%$ RDPS using linear equalization only, single-carrier outperforms OFDM, because the FWM between subcarriers makes OFDM susceptible to nonlinearity. At $100 \%$ RDPS using backpropagation, the performances of single-carrier and OFDM become similar, because dispersion causes both signals to become approximately Gaussiandistributed regardless of the signal profile at transmission. It is observed that using the more accurate IS-SSFM to solve the inverse NLSE despite the receiver not knowing the channel PMD gives better performance than the receiver knowing the PMD but using the less accurate NA-SSFM. As expected, IS-SSFM (including PMD) delivers the best performance, reducing phase error standard deviation by 1 to $3 \mathrm{~dB}$ compared to linear equalization only. 


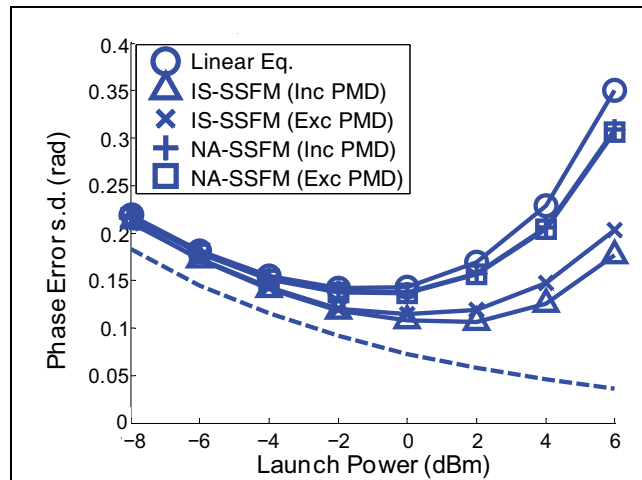

(a)

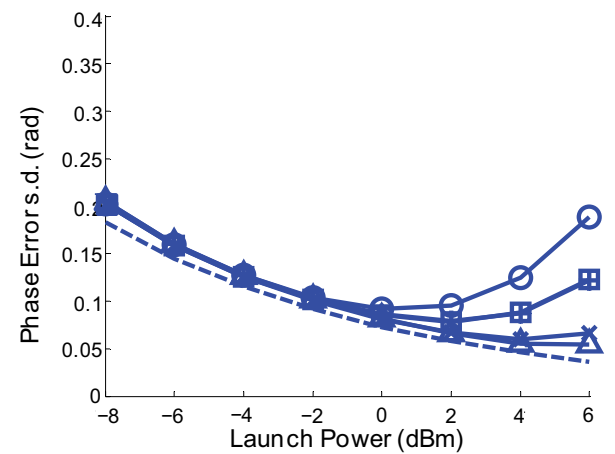

(c)

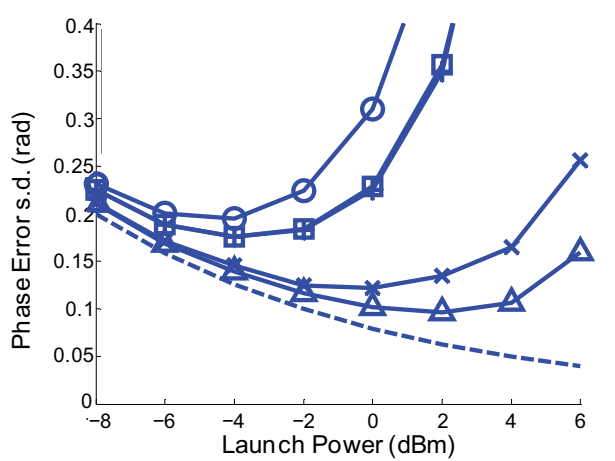

(b)

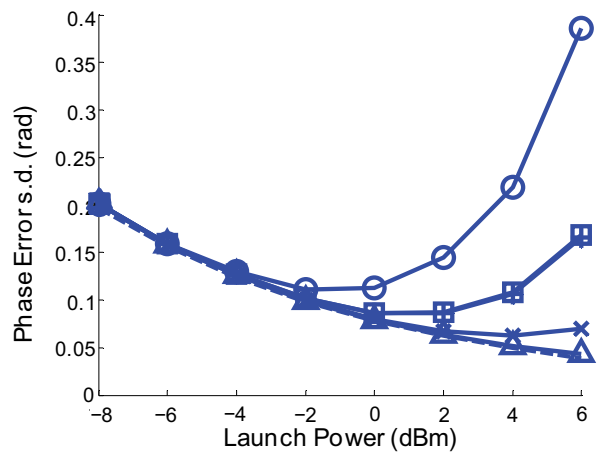

(d)

Fig. 16. System performance for polarization-multiplexed 107-Gb/s transmission through $25 \times 80-\mathrm{km}$ SMF for (a) single-carrier at 0\% RDPS, (b) OFDM at $0 \%$ RDPS, (c) single-carrier at $100 \%$ RDPS, and (d) OFDM at $100 \%$ RDPS.

\section{Further Discussion}

The ability of backpropagation to undo nonlinear effects depends on how accurately it can estimate the signal amplitude profile at every point in the fiber. Noise, PMD, and other distortions not estimated by the receiver, but which change the signal intensity profile, thus degrading performance. Since these effects accumulate with distance, the further a signal is backpropagated, the higher the relative error. In receiver-side backpropagation, the signal intensity profile is known accurately at the receiver, but becomes progressively less accurate as it is traced back to the transmitter. We may therefore expect a small improvement in performance using the setup in Fig. 17, where backpropagation is split evenly between the transmitter and receiver: transmit-side backpropagation inverts the first half of the channel, while receive-side backpropagation inverts the second half. To account for the change in relative error with distance, the parameter $\xi$ should also vary with distance; a larger $\xi$ is used for the spans closer to the transmitter (and receiver), while a smaller $\xi$ is used for spans further away, where the estimated signal intensity is less reliable. 


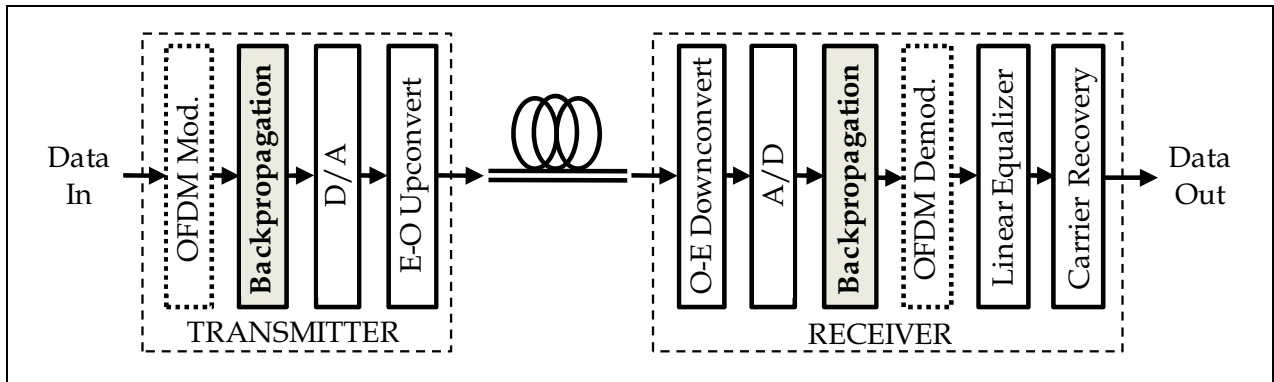

Fig. 17. Backpropagation at transmitter and receiver.

Since backpropagation reduces the effects of nonlinearity, the nonlinear regime of the channel is pushed towards higher launched power (Fig. 18). This has the impact of increasing the optimum launched power and the ultimate achievable capacity. With backpropagation, deterministic nonlinearities do not decrease capacity as they can be mitigated. Furthermore, dispersion-unmanaged transmission causes walkoff between signal and out-of-band noise, reducing their nonlinear interaction. The ultimate limit to capacity therefore arises as a result of nonlinear interaction between signal and in-band noise.

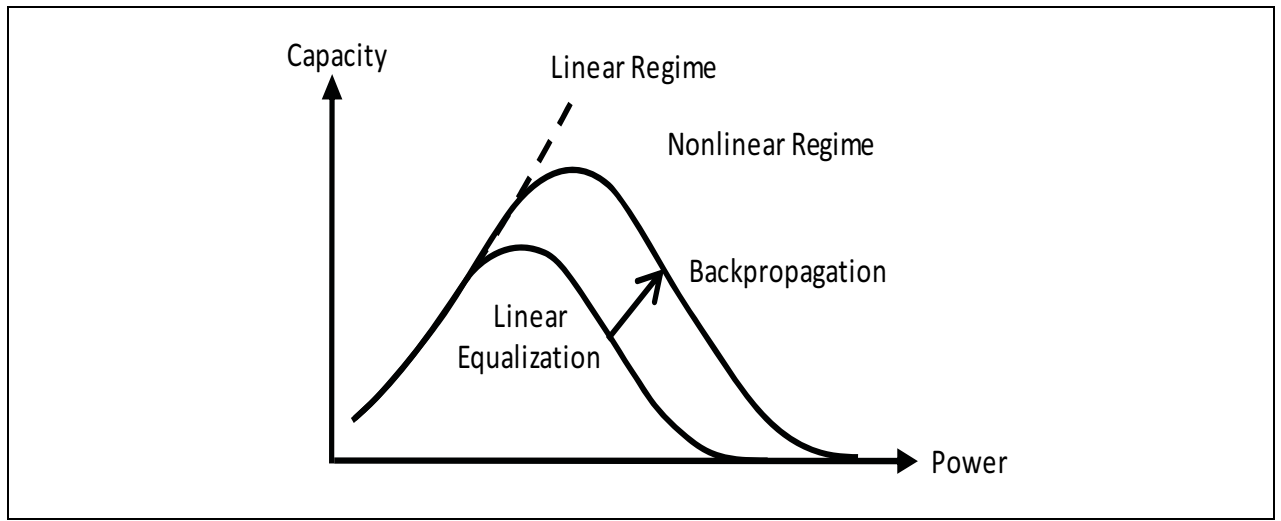

Fig. 18. Effect of backpropagation on capacity.

\section{Conclusion}

Backpropagation is a universal method for jointly compensating linear and nonlinear impairments in an arbitrary channel. The method works independently of the modulation format, and requires finding a numerical solution to the inverse nonlinear Schrödinger equation, which is obtained by reversing the order of the fibers in the link and the signs of the parameters of each fiber. The performance of backpropagation depends on how accurately it estimates the signal amplitude profile at every point in the fiber. Noise, PMD, and other effects not taken into account by the receiver can degrade performance. The best system performance is obtained using dispersion-unmanaged transmission because walkoff reduces nonlinear interaction between signal and out-of-band noise. Interaction between 
signal and in-band noise ultimately limits capacity. Using backpropagation in conjunction with dispersion-unmanaged transmission extends the linear regime of the fiber, enabling higher usable launched power and higher capacity.

\section{References}

Agrawal, G. P. (2001). Nonlinear Fiber Optics 3rd Edition, Academic Press, ISBN 0120451433, San Diego, CA, U.S.A.

Charlet, G.; Maaref, N. ; Renaudier, J. ; Mardoyan, H.; Tran, P. \& Bigo, S. (2006). Transmission of $40 \mathrm{~Gb} / \mathrm{s}$ QPSK with coherent detection over ultra-long distance improved by nonlinearity mitigation, Proceedings of the European Conference on Optical Communication (ECOC 2006), Paper Th4.3.4, Cannes, France, September 2006.

Chowdhury, A \& Essiambre, R.-J. (2004). Optical phase conjugation and pseudolinear transmission, Optics Letters, Vol. 29, No. 10, (May 2004) pp. 1105-1107, ISSN 15394794.

Essiambre, R.-J..; Mikkelsen, B. \& Raybon, G. (1999). Intra-channel cross-phase modulation and four-wave mixing in high-speed TDM, Electronic Letters, Vol. 35, No. 18, (September 1999) pp. 1576-1578, ISSN 0013-5194.

Essiambre, R.-J.; Winzer, P. J.; Qang, X. Q.; Lee, W.; White, C. A. \& Burrows, E. C. (2006). Electronic predistortion and fiber nonlinearity, IEEE Photonic Technology Letters. Vol. 18, No. 17, (September 2006) pp. 1804-1806, ISSN 1041-1135.

Essiambre, R.-J.; Foschini, G. J.; Winzer, P. J.; Kramer, G. \& Burrows, E. C. (2008). The capacity of fiber-optic communication systems, Proceedings of Optical Fiber Communication Conference (OFC 2008), Paper OTuE17, San Diego, CA, U.S.A., March 2008.

Goldfarb, G. \& Li, G. (2009). Efficient backward-propagation using wavelet-based filtering for fiber backward-propagation, Optics Express, Vol. 17, No. 11, (May 2009) pp. 8815-8821, ISSN 1094-4087.

Gordon, J. P. \& Kogelnik, H. (2000). PMD fundamentals: Polarization mode dispersion in optical fibers, Proceedings of the National Academy of Science. Vol. 97, No. 9, (April 2000) pp. 4541-4550, ISSN 0027-8424.

Ho, K.-P. \& Kahn, J. M. (2004). Electronic compensation technique to mitigate nonlinear phase noise. Journal of Lightwave Technology, Vol. 22, No. 3, (March 2004) pp. 779-783, ISSN 0733-8724.

Ip, E.; Lau, A. P. T.; Barros, D. J, \& Kahn, J. M. (2008). Coherent detection in optical fiber systems. Optics Express, Vol. 16, No. 2, (January 2008), pp. 753-791, ISSN 1094-4087.

Ip, E. \& Kahn, J. M. (2008). Compensation of dispersion and nonlinear effects using digital backpropagation, Journal of Lightwave Technology, Vol. 26, No. 20, (October 2008) pp. 3416-3425, ISSN 0733-8724.

Jaworski, M. (2008). Step-size distribution strategies in SSFM simulation of DWDM links, Proceedings of International Conference on Transparent Optical Networks (ICTONMW'08), Paper Fr2A.1, Marrakech, Morocco, December 2008.

Kremp, T. \& Freude, W. (2008). Fast split-step wavelet collocation method for WDM system parameter optimization, Journal of Lightwave Technology, Vol. 23, No. 3, (March 2005) pp. 1491-1502, ISSN 0733-8724. 
Lau, A. P. T.; Rabbani, S. \& Kahn, J. M. (2008). On the statistics of intrachannel four-wave mixing in phase-modulated optical communication systems, Journal of Lightwave Technology, Vol. 26, No. 14, (July 2008) pp. 2128-2135, ISSN 0733-8724.

Leibrich, J. \& Rosenkranz, W. (2003). Efficient numerical simulation of multichannel WDM transmission systems limited by XPM. IEEE Photonics Technology Letters. Vol. 15, No. 3, (March 2003) pp. 395-397, ISSN 1041-1135.

Li, X.; Chen, X.; Goldfarb, G.; Mateo, E.; Kim, I.; Yaman, F. \& Li, G. (2008). Electronic postcompensation of WDM transmission impairments using coherent detection and digital signal processing, Optics Express, Vol. 16, No. 2, (January 2008) pp. 881-888, ISSN 1094-4087.

Liu, X. \& Tkach, R. W. (2009). Joint SPM compensation for inline-dispersion-compensated 112-Gb/2 PDM-OFDM transmission, Proceedings of Optical Fiber Communication Conference (OFC 2009), Paper OTuO5, San Diego, CA, U.S.A., March 2009.

Lowery, A. (2007a). Fiber nonlinearity mitigation in optical links that use OFDM for dispersion compensation, Photonics Technology Letters, Vol. 19, No. 19, (October 2007) pp. 1556-1558, ISSN 1041-1135.

Lowery, A. (2007b). Fiber nonlinearity pre- and post-compensation for long-haul optical links using OFDM, Optics Express, Vol. 15, No. 20, (September 2007) pp. 12965-12970, ISSN 1094-4087.

Mateo, E.; Zhu, L. \& Li, G. (2008). Impact of XPM and FWM on the digital implementation of impairment compensation for WDM transmission using backward propagation, Optics Express. Vol. 16, No. 20, (September 2008), pp. 16124-16137, ISSN 1094-4087.

Mitra, P. P. \& Stark, J. B. (2001). Nonlinear limits to the information capacity of optical fiber communications. Nature, Vol. 411, No. 6841, (June 2001) pp. 1027-1030, ISSN 00280836.

Muslu, G. M. \& Erbay, H. A. (2005). Higher-order split-step Fourier schemes for the generalized nonlinear Schrödinger equation, Mathematics and Computers in Simulation, Vol. 67, No. 6 (January 2005) pp. 581-595, ISSN 0378-4754.

Oppenheim, A. V. \& Schafer, R. W. Discrete-time signal processing, Prentice-Hall, ISBN 013216292X, Upper Saddle River, NJ, U.S.A.

Plura, M.; Kissing, J., Gunkel, M.; Lenge, J.; Elbers, J.-P.; Glingener, C.; Schulz, D. \& Voges, E. (2001). Improved split-step method for efficient fiber simulations, Electronic Letters, Vol. 37, No. 5, (March 2001) pp. 286-287, ISSN 0013-5194.

Rieznik, A. A.; Tolisano, T.; Callegari, F. A.; Grosz, D. F. \& Fragnito, H. L. (2005). Uncertainty relation for the optimization of optical-fiber transmission systems simulations, Optics Express, Vol. 13, No. 10, (May 2005) pp. 3822-3834, ISSN 10944087.

Roberts, K.; Li, C.; Strawczynski, L.; O'Sullivan, M. \& Hardcastle, I. (2006). Electronic precompensation of optical nonlinearity, Photonic Technology Letters, Vol. 18, No. 2, (January 2006) pp. 403-405, ISSN 1041-1135.

Sinkin, O. V.; Holzlöhner, R.; Zweck, J. \& Menyuk, C. (2003). Optimization of the split-step Fourier method in modelling optical-fiber communication systems, Journal of Lightwave Technology, Vol. 21, No. 1, (January 2003) pp. 61-68, ISSN 0733-8724.

Weiss, G. H. \& Maradudin, A. A. (1962). The Baker-Hausdorff formula and a problem in crystal physics, Journal of Mathematical Physics, Vol. 3, No. 4, (July 1962) pp. 771-777, ISSN 0022-2488. 
Zhang, Q. \& Hayee, M. I. (2008). Symmetrized split-step Fourier scheme to control global simulation accuracy in fiber-optic communication systems, Journal of Lightwave Technology, Vol. 26, No. 2, (January 2008) pp. 302-316, ISSN 0733-8724. 


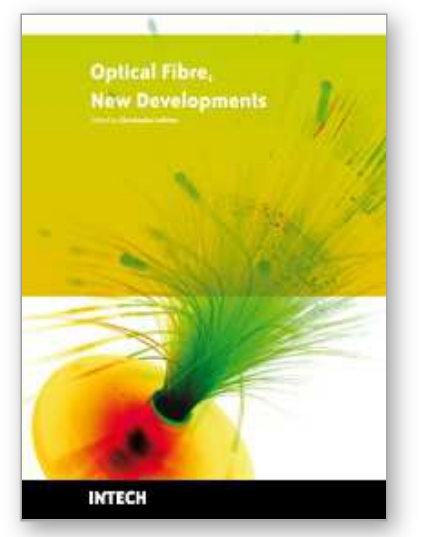

\section{Optical Fiber New Developments}

Edited by Christophe Lethien

ISBN 978-953-7619-50-3

Hard cover, 586 pages

Publisher InTech

Published online 01, December, 2009

Published in print edition December, 2009

The optical fibre technology is one of the hop topics developed in the beginning of the 21 th century and could substantially benefit applications dealing with lighting, sensing and communication systems. Many improvements have been made in the past years to reduce the fibre attenuation and to improve the fibre performance. Nowadays, new applications have been developed over the scientific community and this book fits this paradigm. It summarizes the current status of know-how in optical fibre applications and represents a further source of information dealing with two main topics: the development of fibre optics sensors, and the application of optical fibre for telecommunication systems.

\section{How to reference}

In order to correctly reference this scholarly work, feel free to copy and paste the following:

Ezra Ip and Joseph M. Kahn (2009). Nonlinear Impairment Compensation Using Backpropagation, Optical Fiber New Developments, Christophe Lethien (Ed.), ISBN: 978-953-7619-50-3, InTech, Available from: http://www.intechopen.com/books/optical-fiber-new-developments/nonlinear-impairment-compensation-usingbackpropagation

\section{INTECH}

open science | open minds

\section{InTech Europe}

University Campus STeP Ri

Slavka Krautzeka 83/A

51000 Rijeka, Croatia

Phone: +385 (51) 770447

Fax: +385 (51) 686166

www.intechopen.com

\section{InTech China}

Unit 405, Office Block, Hotel Equatorial Shanghai

No.65, Yan An Road (West), Shanghai, 200040, China

中国上海市延安西路65号上海国际贵都大饭店办公楼 405 单元

Phone: +86-21-62489820

Fax: +86-21-62489821 
(C) 2009 The Author(s). Licensee IntechOpen. This chapter is distributed under the terms of the Creative Commons Attribution-NonCommercial-ShareAlike-3.0 License, which permits use, distribution and reproduction for non-commercial purposes, provided the original is properly cited and derivative works building on this content are distributed under the same license. 